\title{
THE BOUNDARY OF HYPERBOLICITY FOR HÉNON-LIKE FAMILIES
}

\author{
YONGLUO CAO, STEFANO LUZZATTO, AND ISABEL RIOS
}

\begin{abstract}
We consider $C^{2}$ Hénon-like families of diffeomorphisms of $\mathbb{R}^{2}$ and study the boundary of the region of parameter values for which the nonwandering set is uniformly hyperbolic. Assuming sufficient dissipativity, we show that the loss of hyperbolicity is caused by a first homoclinic or heteroclinic tangency and that uniform hyperbolicity estimates hold uniformly in the parameter up to this bifurcation parameter and even, to some extent, at the bifurcation parameter.
\end{abstract}

\section{INTRODUCTION AND STATEMENT OF RESUltS}

Our aim in this paper is to study the boundary of hyperbolicity of certain families of two dimensional maps.

1.1. Hénon-like families. We say that a family of $C^{2}$ plane diffeomorphisms is called a Hénon-like family if it can be written in the form

$$
f_{a, b, \eta}(x, y)=\left(1-a x^{2}+y, b x\right)+\varphi(x, y, a)
$$

where $a \in \mathbb{R}, b \neq 0$ and $\varphi(x, y, a)$ is a $C^{2}$ "perturbation" of the standard Hénon family $h_{a, b}(x, y)=\left(1-a x^{2}+y, b x\right)[$ Hén76] satisfying

$$
\|\varphi\|_{C^{2}(x, y, a)} \leq \eta
$$

In this paper we consider $|b| \neq 0, \eta>0$ fixed sufficiently small and investigate the dynamics as the parameter $a$ is varied. For simplicity we shall therefore omit $b$ and $\eta$ from the notation and denote a Hénon-like family by $\left\{f_{a}\right\}$. For future reference we remark that the inverse of $f_{a}$ is given by an equation of a similar form:

$$
f_{a}^{-1}(x, y)=\left(y / b, x-1+a y^{2} / b^{2}\right)+\tilde{\varphi}(x, y, a)
$$

where $\|\tilde{\varphi}\|_{C^{2}(x, y, a)} \rightarrow 0$ as $\|\varphi\|_{C^{2}(x, y, a)} \rightarrow 0$. We shall suppose without loss of generality that

$$
\|\tilde{\varphi}\|_{C^{2}(x, y, a)} \leq \eta .
$$

Date: February 9, 2005, revised May 10, 2007.

2000 Mathematics Subject Classification. 37D20, 37D25.

IR was partially supported by CAPES, FAPERJ (Brazil) and EPSRC UK. YC was partially supported by NSF(10571130) and NCET of China, the Royal Society and EPSRC UK. SL was partially supported by NSF(10571130) and NCET of China. This work was carried out at Imperial College London and Suzhou University and we acknowledge the hospitality and support of these institutions. We would also like to thank the referee for a careful reading of the paper and several very useful suggestions which have improved the accuracy and presentation of the arguments. 


\subsection{The boundary of hyperbolicity.}

1.2.1. Basic background. Hénon and Hénon-like families have been extensively studied over the last almost 30 years. One of the earliest rigorous results on the subject is [DN79] in which it was shown that the non-wandering set $\Omega_{a, b}$ is uniformly hyperbolic for all $b \geq 0$ and all sufficiently large $a$ (depending on $b$ ). On the other hand, for small $b \neq 0$ and $a \lesssim 2$ there exists positive probability of "strange attractors" which contain tangencies between stable and unstable leaves. This was first proved in [BC91] for the Hénon family and later generalized in [MV93] to Hénon-like families, see also [WY01, LV03]. These attractors cannot be uniformly hyperbolic due to the presence of tangencies but turn out to satisfy weaker nonuniform hyperbolicity conditions [BY93, BY00, BV01].

1.2.2. Complex methods. More recently Bedford and Smillie have described the transition between these two regimes for Hénon families by identifying and describing some of the properties of the boundary of uniform hyperbolicity [BS06]. In particular they show that for small $|b|$, the nonwandering set is uniformly hyperbolic up until the first parameter $a$ at which a tangency occurs between certain stable and unstable manifolds. Combining this with the statements contained in [BS02] their results also imply uniform bounds on the Lyapunov exponents of all invariant probability measures at the bifurcation parameter [Bed05]. Their methods rely crucially on previous work [BS04] which in turn is based on the polynomial nature of the Hénon family, a feature which allows them to consider its complexification and to apply original and highly sophisticated arguments of holomorphic dynamics.

1.2.3. Real methods. In this paper we develop a new and completely different strategy to the problem, based purely on geometric "real" arguments, which have the advantage of applying to general $C^{2}$ Hénon-like families. We also obtain the analogous uniformity results by showing that the hyperbolicity expansion and contraction rates are uniform right up to the point of tangency and that even at the point of tangency some strong version of nonuniform hyperbolicity continues to hold: all Lyapunov exponents of all invariant measures are uniformly bounded away from 0 .

Theorem 1. For all $|b|>0$ and $\eta>0$ sufficiently small we have the following property. For every Hénon-like family $\left\{f_{a}\right\}_{a \in \mathbb{R}}$ of plane diffeomorphisms there exists a unique $a^{*}$ such that

(1) For all $a>a^{*}$ the nonwandering set $\Omega_{a}$ is uniformly hyperbolic;

(2) For $a=a^{*}$ the nonwandering set $\Omega_{a^{*}}$ contains an orbit of tangency but is "almost uniformly hyperbolic" in the sense that all Lyapunov exponents of all invariant probability measures supported on $\Omega$ are uniformly bounded away from 0.

Moreover, the bounds on the expansion and contraction rates for all $a \geq a^{*}$ are independent of $a$ and of the family. 
1.2.4. Singular perturbations. We remark that this is not the only existing definition of Hénon-like in the literature. One standard approach is to consider "singular" perturbations of the limiting one-dimensional map corresponding to the case $b=0$ :

$$
f_{a}(x, y)=\left(1-a x^{2}, 0\right)+\varphi_{a}(x, y) .
$$

This formulation however has some slight technical issues. For example, one cannot assume that $\left\|\varphi_{a}\right\|_{C^{2}}$ is small on all of $\mathbb{R}^{2}$ since that would violate the requirement that $f_{a}$ be a global diffeomorphism of $\mathbb{R}^{2}$. This can be dealt with by restricting our attention to some compact region, say $[-2,2] \times[-2,2]$, and supposing only that $\left\|\varphi_{a}\right\|_{C^{2}} \leq \eta$ in this region. Our arguments apply in this case also and yield a more local result on the hyperbolicity of the nonwandering set restricted to $[-2,2] \times[-2,2]$.

\subsection{Basic definitions.}

1.3.1. Nonwandering set. We recall that a point $z$ belongs to the nonwandering set $\Omega$ of $f$ if it has the property that for every neighbourhood $\mathcal{U}$ of $z$ there exists some $n \geq 1$ such that $f^{n}(\mathcal{U}) \cap \mathcal{U} \neq \emptyset$. The nonwandering set is always invariant and closed (and thus if bounded, also compact).

1.3.2. Uniform hyperbolicity. We say that a compact invariant set $\Omega$ is uniformly hyperbolic (with respect to $f$ ) if there exists constants $C^{u}, C^{s}>0, \lambda^{u}>0>\lambda^{s}$ and a continuous decomposition $T \Omega=E^{s} \oplus E^{u}$ of the tangent bundle such that for every $x \in \Omega$, every non-zero vector $v^{s} \in E_{z}^{s}$ and $v^{u} \in E_{z}^{u}$ and every $n \geq 1$ we have

$$
\left\|D f_{z}^{n}\left(v^{s}\right)\right\| \leq C^{s} e^{\lambda^{s} n} \text { and }\left\|D f_{z}^{n}\left(v^{u}\right)\right\| \geq C^{u} e^{\lambda^{u} n} .
$$

By standard hyperbolic theory, the stable and unstable subspaces $E_{z}^{s}, E_{z}^{u}$ are tangent everywhere to the stable and unstable manifolds. In particular uniform hyperbolicity is incompatible with the presence of any tangencies in $\Omega$ between any stable and any unstable invariant manifolds associated to points of $\Omega$.

1.3.3. Nonuniform hyperbolicity. A weaker notion of hyperbolicity can be formulated in terms of invariant measures. For simplicity we restrict our discussion to the two-dimensional setting, as relevant to the situation we consider in this paper. Let $\mu$ be an $f$-invariant ergodic probability measure with support in some compact invariant set $\Omega$. By Oseledec's Ergodic Theorem [Ose68] there exist constants $\lambda^{u} \geq \lambda^{s}$ and a measurable decomposition $T \Omega=E^{s} \oplus E^{u}$ such that for $\mu$-almost every $z$ and every non-zero vector $v^{s} \in E_{z}^{s}$ and $v^{u} \in E_{z}^{u}$ we have

$$
\lim _{n \rightarrow \infty} \frac{1}{n} \log \left\|D f_{z}^{n}\left(v^{s}\right)\right\|=\lambda^{s} \quad \text { and } \quad \lim _{n \rightarrow \infty} \frac{1}{n} \log \left\|D f_{z}^{n}\left(v^{u}\right)\right\|=\lambda^{u} .
$$

The constants $\lambda^{s}$ and $\lambda^{u}$ are called the Lyapunov exponents associated to the measure $\mu$. We say that $\mu$ is hyperbolic [YP76, Pes77] if

$$
\lambda^{u}>0>\lambda^{s} .
$$


Clearly (1) implies (2) for any $\mu$. The converse however is false in general: the measurable decomposition may not extend to a continuous one on all of $\Omega$ and the exponential expansion and contraction in (2) implies only a limited version of (1) in which the constants $C^{s}, C^{u}$ are measurable functions of $x$ and not bounded away from 0 . This definition of hyperbolicity in terms of Lyapunov exponents is sometimes called nonuniform hyperbolicity and is consistent in principle with the existence of tangencies between stable and unstable manifolds.

1.3.4. The boundary between uniform and nonuniform hyperbolicity. In general there may be many ergodic invariant probability measures supported in $\Omega$ of which some may be hyperbolic and some not. Even if they are all hyperbolic the corresponding Lyapunov exponents may not be uniformly bounded way from 0 . The situation in which all Lyapunov exponents of all ergodic invariant measures are uniformly bounded away from zero is, in some sense, as "uniformly hyperbolic" as one can get while admitting the existence of tangencies. This situation can indeed occur, for example in the present context of Hénonlike maps. A first example of a set satisfying this property was given in [CLR06].

1.4. A one-dimensional version. After completing the proof of the Theorem 1 we realized that much simpler versions of our arguments yield an analogous, new and non-trivial, result in the context of one-dimensional maps. We explain and give a precise formulation of this result. We consider first the quadratic family

$$
h_{a}(x)=1-a x^{2} .
$$

We choose this particular parametrization for convenience and consistency with our two dimensional results, but any choice of smooth family of unimodal or even multimodal maps with negative Schwarzian derivative would work in exactly the same way. It is well known that for $a>2$ the nonwandering set $\Omega_{a}$ is uniformly expanding although we emphasize here that this depends crucially on the negative Schwarzian derivative property. The negative Schwarzian property is not robust with respect to $C^{2}$ perturbations and standard methods do not therefore yield this statement for such perturbations.

Theorem 2. There exists a constant $\eta>0$ such that if a family $\left\{g_{a}\right\}$ of $C^{2}$ one-dimensional maps satisfies

$$
\left\|g_{a}-h_{a}\right\|_{C^{2}} \leq \eta
$$

then there exists a unique parameter value $a^{*}$ such that

(1) For all $a>a^{*}$ the non-wandering set $\Omega$ is uniformly hyperbolic;

(2) For $a=a^{*}$ the Lyapunov exponents of all ergodic invariant probability measures are all positive and uniformly bounded away from 0.

Moreover the rates of expansion and the bound on the Lyapunov exponents are uniform, independent of the family and of the parameter.

The proof of this result is exactly the same as that of Theorem 1 but hugely simpler as all more geometrical arguments concerning curvature etc become essentially trivial. 
We emphasize that the uniform expansivity of $\Omega_{a}$ for a particular parameter value $a>2$ is of course robust under sufficiently small perturbations of $f_{a}$, by standard hyperbolic theory. However this approach requires the size of the perturbation to depend on the parameter $a$ and in particular to shrink to zero as $a$ tends to 2 . The crucial point of our approach, both in this one-dimensional setting, as in the two-dimensional setting is that the size of the perturbation does not depend on the parameter.

1.5. Overview of the paper. We have divided our argument into three main sections. In Section 2 we analyze the geometric structure of stable and unstable manifolds of the two fixed points and define the parameter $a^{*}$ as the first parameter for which a tangency occurs between some compact parts of these manifolds. We also identify a region $\mathcal{D}$ which we show contains the non-wandering set. In Section 3 we define a "critical neighbourhood" $\Delta_{\varepsilon}$ outside of which our maps are uniformly hyperbolic by simple perturbation arguments. However $\Delta_{\varepsilon}$ does contain points of $\Omega$ and thus we cannot ignore this region. To control the hyperbolicity in $\Delta_{\varepsilon}$ we introduce the notions of Hyperbolic Coordinates and Critical Points which form the key technical core of our approach. Finally, in Section 4 we apply these techniques to prove the required hyperbolicity properties.

\section{ThE NON-WANDERING SET}

In this section we define the parameter $a^{*}$ as in the statement of our main Theorem, and show that for $a \geq a^{*}$ the nonwandering set is contained in the closure if the unstable manifold of a hyperbolic fixed point restricted to a certain compact region of $\mathbb{R}^{2}$.

2.1. The parameter $a^{*}$. We define the bifurcation parameter $a^{*}$ below as the first parameter for which there is a tangency between certain compact parts of the stable and unstable manifolds of the fixed points. This does not immediately imply that it is a first parameter of tangency though this will follow from our proof of the fact that the nonwandering set is uniformly hyperbolic for all $a>a^{*}$.

2.1.1. Fixed points and invariant manifolds for the one-dimensional limit. For the endomorphisms $h_{a}=h_{a, 0}$ with $a \geq 2$, there are two fixed points,

$$
p_{a}=\frac{-1+\sqrt{1+4 a}}{2 a}>q_{a}=\frac{-1-\sqrt{1+4 a}}{2 a}
$$

both hyperbolic. For the special parameter value $a=2$, to simplify the notation below, we write

$$
f_{*}=h_{2,0} \text {, and denote the two fixed points by } p_{*}=(1 / 2,0) \text { and } q_{*}=(-1,0) \text {. }
$$

Since $q^{*}$ and $p^{*}$ are repelling in the horizontal direction, their stable sets are simply their preimages:

$$
W^{s}\left(q^{*}\right)=\bigcup_{n \geq 0} f_{*}^{-n}\left(q^{*}\right) \quad \text { and } \quad W^{s}\left(p^{*}\right)=\bigcup_{n \geq 0} f_{*}^{-n}\left(p^{*}\right) .
$$

In particular these sets contain the following curves

$$
f_{*}^{-1}\left(q_{*}\right)=\left\{(x, y): f_{*}(x, y)=\left(1-2 x^{2}+y, 0\right)=(-1,0)\right\}=\left\{y=2 x^{2}-2\right\}
$$




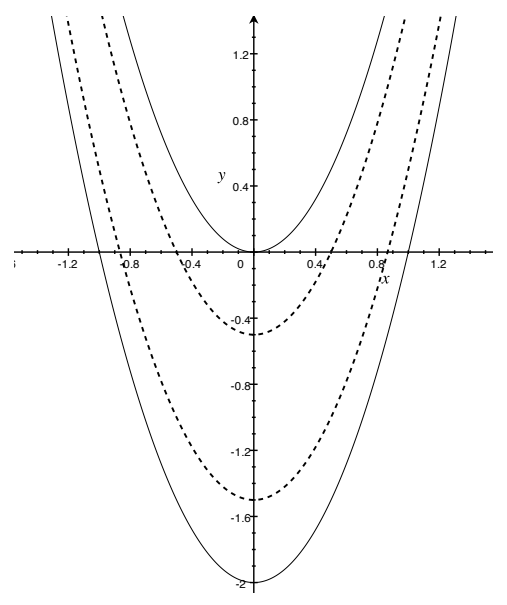

FiguRE 1. First two "generations" of $W^{s}\left(q_{*}\right)$ and $W^{s}\left(p_{*}\right)$.

and

$$
f_{*}^{-2}\left(q_{*}\right)=\left\{(x, y): f_{*}(x, y)=\left(1-2 x^{2}+y, 0\right)=(1,0)\right\}=\left\{y=2 x^{2}\right\}
$$

The first preimage of $q^{*}$ is a parabola with a minimum at $(0,-2)$ and intersecting the $x$-axis at $x= \pm 1$, and having slope equal to -4 at the point $q_{*}=(-1,0)$ and the second is a parabola with a minimum at $(0,0)$. Similarly we can compute

$$
f_{*}^{-1}\left(p_{*}\right)=\left\{z=(x, y): f_{*}(z)=\left(1-2 x^{2}+y, 0\right)=(1 / 2,0)\right\}=\left\{y=2 x^{2}-1 / 2\right\}
$$

which is a parabola with a minimum at $(0,-1 / 2)$, intersecting the $x$-axis at $x= \pm 1 / 2$ and having slope equal to 2 at the point $p_{*}=(1 / 2,0)$, and

$$
f_{*}^{-2}\left(p_{*}\right)=\left\{z=(x, y): f_{*}(z)=\left(1-2 x^{2}+y, 0\right)=(-1 / 2,0)\right\}=\left\{y=2 x^{2}-3 / 2\right\}
$$

which is a parabola with a minimum at $(0,-3 / 2)$.

The unstable manifolds going $W^{u}\left(q_{*}\right)$ and $W^{u}\left(p_{*}\right)$ can be defined and computed in a similar way and are easily seen to be horizontal.

2.1.2. Fixed points for Hénon-like families. Consider first the Hénon family $h_{a, b}(x, y)=$ $\left(1-a x^{2}+y, b x\right)$. For $b \neq 0, h_{a, b}$ is a diffeomorphism. The hyperbolicity of the fixed points implies that there exists a neighbourhood of the set $\{(a, 0): a \geq 2\}$ corresponding to pairs of parameters for which there is an analytic continuation $q_{a, b}, p_{a, b}$ as hyperbolic fixed points of $h_{a, b}$. Considering $\eta$ small, we also have that the analytic continuations $q_{f_{a}}$ and $p_{f_{a}}$ are also well defined and hyperbolic. For simplicity we shall often just refer to these two points as $q, p$ leaving implicit their dependence on $f$.

Explicit formulas for $q_{a, b}, p_{a, b}$ can be easily derived from the equation $\left(1-a x^{2}+y, b x\right)=$ $(x, y)$ but these would not be particular useful. Instead we just observe that the fixed points must lie on the line $\{y=b x\}$ and so in particular this means that for $a \approx 2$ and $b \gtrsim 0$ the vertical coordinates of $q_{a, b}$ and $p_{a, b}$ are negative and positive respectively, and the converse for $b \lesssim 0$. Clearly the same holds for $q=q_{f_{a}}$ and $p=p_{f_{a}}$ if $\eta$ is sufficiently 
small. Moreover, the determinant of $h_{a, b}$ is given by

$$
\operatorname{det} D h_{a, b}=\operatorname{det}\left(\begin{array}{cc}
-2 a x & 1 \\
b & 0
\end{array}\right)=-b .
$$

In particular the determinant is constant and negative if $b$ is positive and positive if $b$ is negative. We thus refer to the case $b>0$ as the orientation-reversing case, and the case $b<0$ as the orientation-preserving case. Recall that the determinant of a matrix
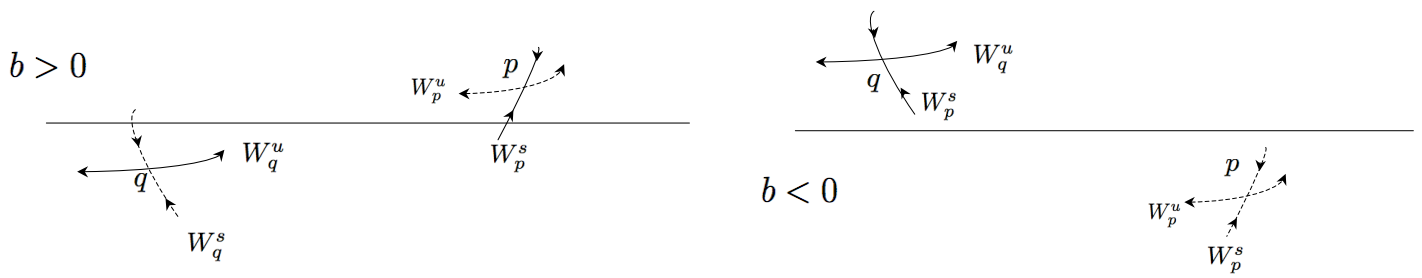

Figure 2. Fixed points and their local stable and unstable manifolds for the orientation-reversing $(b>0)$ and the orientation-preserving $(b<0)$ case (dotted curves indicate negative eigenvalues)

is the product of the eigenvalues, and thus in particular, the sign of the determinant has implications for the sign of the eigenvalues which, as we shall see, in turn has implications for the geometry of the stable and unstable manifolds of the fixed points. For $b=0$ the fixed points $p_{*}$ and $q_{*}$ have derivatives 4 and -2 respectively, and thus, for $b \neq 0$ and $\eta$ small, the expanding eigenvalues of $p$ and $q$ are $\approx 4$ and $\approx-2$ respectively. This implies that for $b \gtrsim 0$, the orientation-reversing case, the contracting eigenvalues of $q$ and $p$ must be $<0$ and $>0$ respectively, while for $b<0$, the orientation preserving case, they must be $>0$ and $<0$ respectively. The two situations are illustrated in Figure 2 with dashed lines showing the invariant manifolds corresponding to negative eigenvalues.

2.1.3. Analytic continuation of stable and unstable manifolds. By classical hyperbolic theory, compact parts of the stable manifolds depend continuously on the map (see e.g. [PdM82]). Therefore, for small $b$ and small $\eta$ the analytic continuations $q, p$ of the fixed points $q_{*}$ and $p_{*}$ have stable and unstable manifolds which are close to those computed above for the limiting case. Elementary calculations show that the actual geometrical relations between these continuations depend on whether we consider the orientation reversing $(b>0)$ or the orientation preserving $(b<0)$ case, and are as illustrated in Figure 3 . We let

$$
\Gamma_{a}^{u}(p) \subset W_{a}^{u}(p), \quad \Gamma_{a}^{s}(q) \subset W_{a}^{u}(q), \quad \Gamma_{a}^{u}(q) \subset W_{a}^{u}(q)
$$

denote the compact parts of the stable and unstable manifold as shown in Figure 3 and notice that, in particular, since for $b=0$ and $a>2$ the unstable manifold of $p_{a}$ and $q_{a}$ extend to the whole of the line, for each $a>2$ and $b>0$ sufficiently small we have that $W_{l o c}^{u}(p)$ crosses $W_{l o c}^{s}(p)$ four times, and for each $a>2$ and $b<0$ sufficiently small we have that $W_{l o c}^{u}(q)$ crosses $W_{l o c}^{s}(q)$ four times, and also we can ensure that the compact parts defined above and in the Figure intersect transversally. Again this continues to hold also for a Hénon-like family for sufficiently small $\eta$. 


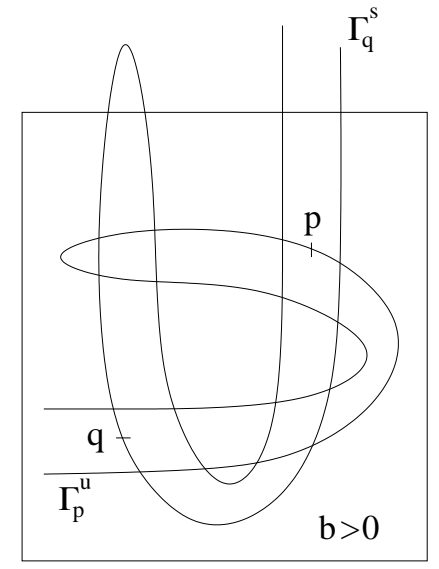

(a)

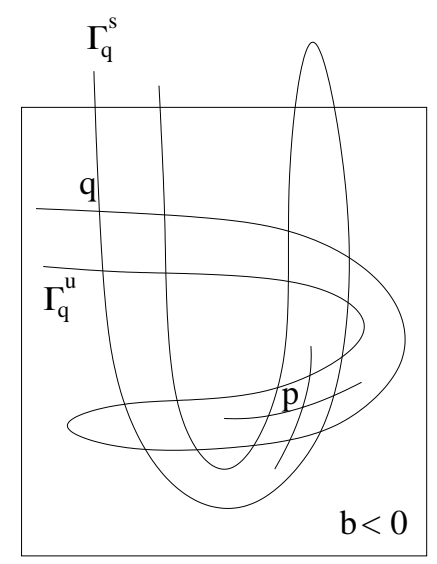

(b)

FIGURE 3. Invariant manifolds for $a>a^{*}$

2.1.4. Definition of $a^{*}$. We are now ready to define the parameter $a^{*}$. We fix $b \neq 0$. For an orientation-reversing $(b>0)$ Hénon-like family $f_{a}$, we let

$$
a^{*}=\inf \left\{a: \Gamma_{a}^{s}(p) \text { and } \Gamma_{a}^{u}(q) \text { intersect transversally }\right\} .
$$

For an orientation-preserving $b<0$ Hénon-like family $f_{a}$, we let

$$
a^{*}=\inf \left\{a: \Gamma_{a}^{s}(q) \text { and } \Gamma_{a}^{u}(q) \text { intersect transversally }\right\} .
$$

We also define a parameter

$$
\hat{a}<a^{*}
$$

as the inf of parameters $a$ for which $W_{l o c}^{u}(p)$ crosses $W_{l o c}^{s}(p)$ four times $(b>0)$ or $W_{l o c}^{u}(q)$ crosses $W_{l o c}^{s}(q)$ four times $(b<0)$. Clearly this is a weaker condition and thus $a^{*} \geq \hat{a}$. Notice that $a^{*}$ and $\hat{a}$ converge to $a=2$ as $b$ and $\eta$ tend to 0 .

2.2. Localization of the nonwandering set. In this section we carry out a detailed geometrical study aimed at showing that the nonwandering set is contained in a relatively restricted region. To prove hyperbolicity we will then just have to focus our efforts in this region. For the moment we restrict ourselves to the orientation-reversing case. At the end we shall remark how the orientation-preserving case follows by identical arguments with a few minor changes of notation. First of all we let $\mathcal{D}$ denote the closed topological disc bounded by compact pieces of the $W^{u}(p)$ and $W^{s}(q)$ as shown in Figure 4. The main result of this section is the following

Proposition 1. For all $a>\hat{a}$ we have

$$
\Omega \subset \overline{W^{u}(p)} \cap \mathcal{D} \cap\{[-2,2] \times(-4 b, 4 b)\} .
$$

Remark. In this paper we are interested in parameters $a \geq a^{*}(\geq \hat{a})$, but it is worth observing that if follows from Proposition 1 that for all $a \in\left(\hat{a}, a^{*}\right)$, and so in particular for a certain range of parameter values which may contain multiple tangencies the recurrent dynamics is captured to some extent by the dynamics on $W^{u}(p)$. This includes in particular 


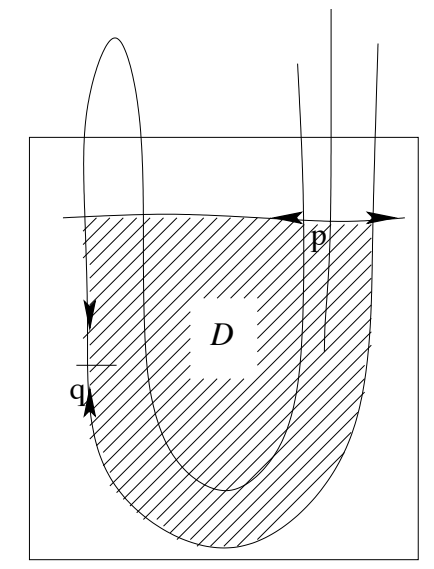

Figure 4. The region $\mathcal{D}$

all complex dynamical phenomena associated to the unfolding of the tangency at the parameter $a^{*}$ (indeed, this includes the range of parameters considered by BenedicksCarleson in [BC91].

We split the proof of Proposition 1 in several Lemmas. Once again we deal first with the case $b>0$. At the end of the proof we indicate the necessary modifications in order to deal with the case $b<0$. We first define a relatively "large" region $R$ and show that $\Omega \subset R$ and then show in separate arguments that $\Omega \subset \mathcal{D}$ and $\Omega \subset \overline{W^{u}(p)}$, and finally refine our estimate to obtain the statement in the Proposition. Let

$$
R=(-2,2) \times(-4,4 b) \subset \hat{R}=(-2,2) \times(-4,2)
$$

We also define the following 6 (overlapping) regions (see Figure 5):

$$
\begin{aligned}
& V_{1}=\{(x, y): x \leq-2, y \leq|x|\}, \\
& V_{2}=\{(x, y): x \leq 2, y \leq-4\}, \\
& V_{3}=\{(x, y): x \geq 2, y \leq 2\}, \\
& V_{4}=\{(x, y): x \geq-2, y \geq 2\}, \\
& V_{5}=\{(x, y): x \leq-2, y \geq|x|\}, \\
& V_{6}=\{(x, y):|x| \leq 2, y \geq 4 b\}
\end{aligned}
$$

Then

$$
\hat{R}=\mathbb{R}^{2} \backslash\left(V_{1} \cup \cdots \cup V_{5}\right) \text { and } R=\mathbb{R}^{2} \backslash\left(V_{1} \cup \cdots \cup V_{6}\right)
$$

We prove the following two statements.

Lemma 1. $\Omega \subset R$.

Proof. We show that the orbit of every point $(x, y) \in V_{i}, i=1, \ldots, 6$ is unbounded in either backward or forward time. This implies in particular that no such point is nonwandering. For $n \in \mathbb{Z}$, let $\left(x_{n}, y_{n}\right)=f_{a}^{n}(x, y)$. We shall use repeatedly the fact that $a \approx 2$ and $b \approx 0$. 

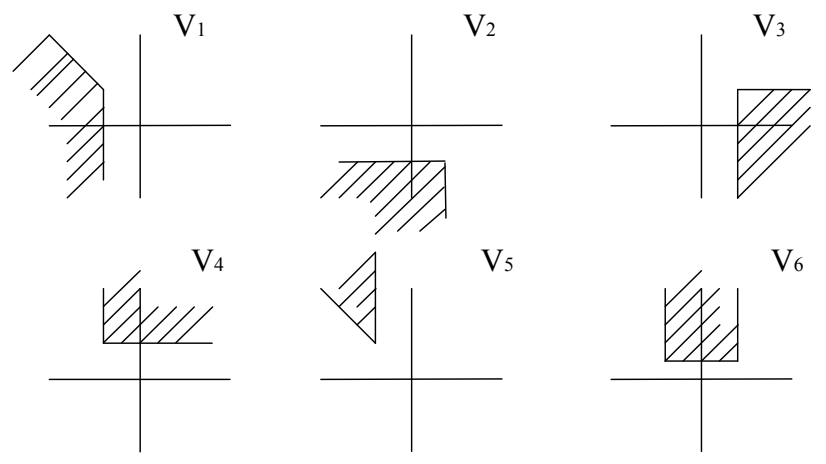

FiguRe 5. Regions $V_{1}$ to $V_{6}$

For $(x, y) \in V_{1}$ we have $x \leq-2$ and therefore $x_{1}=1-a x^{2}+y+\varphi_{1}(x, y, a) \leq 1-a x^{2}+$ $|x|+\eta \leq-2$ and $y_{1}=b x+\varphi_{2}(x, y, a) \leq-2 b+\eta<\left|x_{1}\right|$, as long as $\eta$ is sufficiently small. Thus $\left(x_{1}, y_{1}\right) \in V_{1}$, and $\left|x_{1}\right| \geq a x^{2}-|x|-1-\eta \geq 2|x|$. Repeating the calculation we have $\left|x_{n}\right| \geq 2^{n}|x|$ and so $\left|x_{n}\right| \rightarrow \infty$.

For $(x, y) \in V_{2}$ we have $x_{1}=1-a x^{2}+y+\varphi_{1}(x, y, a) \leq-2$ and $y_{1}=b x+\varphi_{2}(x, y, a) \leq$ $2 b+\eta<\left|x_{1}\right|$. Thus $\left(x_{1}, y_{1}\right) \in V_{1}$ and so $\left|x_{n}\right| \rightarrow \infty$.

Similarly, for $(x, y) \in V_{3}$ we have $x_{1}=1-a x^{2}+y+\varphi_{1}(x, y, a) \leq 1-2 \cdot 2^{2}+2+\eta \leq-2$ and $y_{1}=b x+\varphi_{2}(x, y, a) \leq 2 b+\eta<\left|x_{1}\right|$. Thus $\left(x_{1}, y_{1}\right) \in V_{1}$ and we argue as above.

For $(x, y) \in V_{4}$ we consider backward iterations of $f_{a}$. Note that $\left(x_{-1}, y_{-1}\right)=(y / b, x-$ $\left.1+a y^{2} / b^{2}\right)+\tilde{\varphi}(x, y, a)$. Then $x_{-1} \geq 2 / b-\eta \geq-2$ and $y_{1} \geq-2+4 a / b^{2}-\eta \geq 2$. Thus $f^{-1}(x, y) \in V_{4}$ and $y_{1} \geq y / b$. Therefore $y_{-n} \geq y / b^{n}$ and so $\left|y_{-n}\right| \rightarrow \infty$.

For $(x, y) \in V_{5}$ we have $y \geq|x| \geq 2$. Thus $x_{1} \geq y / b-\eta \geq 2$ and $y_{1} \geq y^{2} / b^{2} \geq 2$. So we have that $f^{-1}(x, y) \in V_{4}$, and we argue as above.

For $(x, y) \in V_{6}$, we have $x_{-1}=y / b+\tilde{\varphi}_{2}(x, y, a) \geq 2$ and $y_{-1} \geq 2$. Therefore, $f_{a}(x, y) \in V_{4}$ and again, we argue as above.

Lemma 2. $\mathcal{D} \subset \hat{R}$.

Proof. The arguments used above have implications for the locations of the stable and unstable manifolds of the fixed points. Indeed the stable manifolds of the fixed points cannot intersect $V_{1} \cup V_{2} \cup V_{3}$ since all points in this region tend to infinity in forward time, whereas, by definition, points in the stable manifolds tend to the fixed points under forward iteration. Similarly the unstable manifolds of the fixed points cannot intersect $V_{4} \cup V_{5}$ since all points in this region tend to infinity in backwards time. By definition $\mathcal{D}$ is bounded by arcs of stable and unstable manifolds of the fixed point as in the Figure and therefore $\mathcal{D} \subset \mathbb{R}^{2} \backslash\left(V_{1} \cup \ldots \cup V_{5}\right)=(-2,2) \times(-4,2)$.

Lemma 3. $\Omega \subset \mathcal{D}$.

Proof. To show that $\Omega \subset \mathcal{D}$ we refine the strategy used in the proof of the previous lemma, and show that the orbits of all points outside $\mathcal{D}$ are unbounded in either backward or 

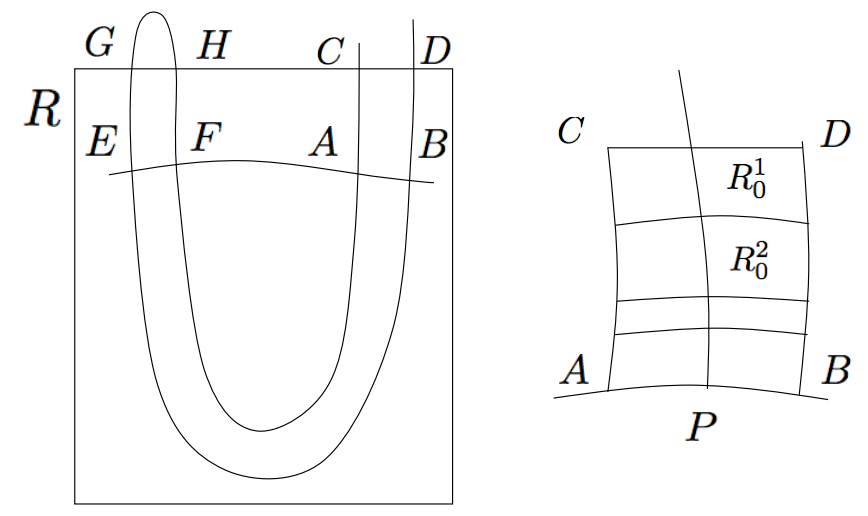

FiguRE 6. Regions $R_{0}^{i}$

forward time. Since we have already shown that $\Omega \subset R$, we need to consider only points in the region $R \backslash \mathcal{D}$.

Subdividing. We write

$$
R \backslash \mathcal{D}=R_{0} \cup R_{1} \cup R_{2} \cup R_{3}
$$

where the regions $R_{0}, R_{1}, R_{2}, R_{3}$ are defined as follows. Consider the points $A$ and $B$ of intersection of $W^{u}(P)$ and $W^{s}(q)$ and $C, D$ of intersection of $W^{s}(q)$ and $y=4 b$ as in the Figure 6 . We let $R_{0}$ denote the closed region bounded by the arcs of manifold $A C$, $A B$ and $B D$, and the segment $C D$. We let $R_{1}$ denote the region bounded by the arc of manifolds $H F, F A$ and $A C$, and the segment $H C$. We let $R_{2}$ denote the region bounded by the arcs of $W^{u}(p)$ and $W^{s}(q)$ between the points $E$ and $F$, as in Figure 6 . We let $R_{3}=R \backslash\left(\mathcal{D} \cup R_{0} \cup R_{1} \cup R_{2}\right)$. We also define

$$
\tilde{R}_{3} \subset R_{3}
$$

as the region satisfying $-2 b-\eta<y<2 b+\eta$ at the left side of the arc of $W^{s}(q)$ between the points $I$ and $J$, of intersection of that manifold with the lines $y=-2 b-\eta$ and $y=2 b+\eta$, as in the Figure 7 (b).

Points of $R_{0}$ escape in backward time. Since $b$ is small, we have that all the points $(x, y) \in$ $R_{0}$ satisfy $x>0.2$. Notice that, for the unperturbed Hénon map $h_{a, b}(x, y)=\left(1-a x^{2}+\right.$ $y, b x)$, we have that any piece of curve $\gamma$ with slope less than $1 / 10$ contained in the region where $|x|>0.2$ is mapped to another curve with slope less than $1 / 10$. Indeed, letting $\left(v_{1}, v_{2}\right)$ denote a tangent vector to $\gamma$ with $\left|v_{2}\right| /\left|v_{1}\right|<1 / 10$, we have $\left(v_{1}^{\prime}, v_{2}^{\prime}\right)=D h_{a, b}\left(v_{1}, v_{2}\right)=$ $\left(-2 a x v_{1}+v_{2}, b v_{1}\right)$ whose slope is $\left|v_{2}^{\prime}\right| /\left|v_{1}^{\prime}\right|=\left|b /\left(-2 a x+\left(v_{2} / v_{1}\right)\right)\right|<1 / 10$, provided $b$ is small and $a$ is close to 2 . For future reference, notice that, if $|x|>0.5$, we also have that the norm of $\left(v_{1}, v_{2}\right)$ is uniformly expanded. So, since $f_{a}$ is close to $h_{a, b}$ in the $C^{2}$ topology, we can assume that $f_{a}$ also has this property in $R_{0}$.

Now call $\alpha_{n}$ the successive images of the segment $C D$ intersected to $R_{0}$. Since they cannot intersect each other, and $C D$ has a point of the stable manifold of $p$, the curves $\alpha_{n}$ determine a system of "fundamental domains" in $R_{0}$ : they cross $R_{0}$ from one stable 

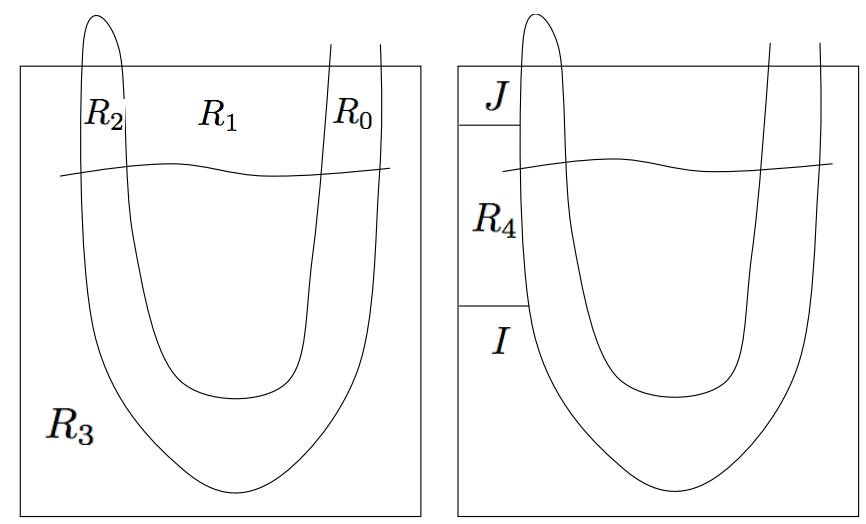

FiguRe 7 . Regions $R_{1}$ to $R_{4}$

boundary to the other, and they converge to the arc of unstable manifold $A B$. Call $R_{0}^{i}$ the region of $R_{0}$ between $\alpha_{i-1}$ and $\alpha_{i}, \alpha_{0}=C D$, and notice that $f^{-1}\left(R_{0}^{i}\right) \subset R_{0}^{i-1}$. We also have that $f^{-1}\left(R_{0}\right)$ falls outside $R$. That implies that $R_{0} \backslash A B$ does not intersect $\Omega$, and any point which has an iterate in $R_{0} \backslash A B$ is not in $\Omega$.

Points of $R_{1}$ map to $R_{3}$. We show that $f\left(R_{1}\right) \cap R \subset R_{3}$. Indeed, the unstable eigenvalue of $p$ is positive and therefore $f\left(R_{1}\right)$ must remain on the same side of $W^{s}(q)$ as $R_{1}$. Also, since $f(R) \subset \mathbb{R} \times[-2 b-\eta, 2 b+\eta]$ we have that $f\left(R_{1}\right)$ does not intersect any of $\mathcal{D}, R_{0}, R_{1}, R_{2}$.

Points of $R_{3}$ map to $\tilde{R}_{3}$. We now show that $f\left(R_{3}\right) \subset \tilde{R}_{3}$. Again, we use the fact that $f(R) \subset \mathbb{R} \times[-2 b-\eta, 2 b+\eta]$. Then, since one of the components of the boundary of $R_{3}$ is an arc of stable manifold of $q$ containing the fixed point $q$, and the unstable eigenvalue of $q$ is positive, we conclude that the image of $R_{3}$ is contained $\tilde{R}_{3}$.

Points of $\tilde{R}_{3}$ escape in forward time. We can assume, if $b$ is small, that all the points $(x, y)$ in $\tilde{R}_{3}$ satisfy $x<-0.5$ (notice that, for $b=0$, we have $q=(-1,0)$. Take $t$ a point in $\tilde{R}_{3} \backslash W^{s}(q)$, and connect $t$ to the boundary of $\tilde{R}_{3}$ through a horizontal line inside $\tilde{R}_{3}$, determining a point $t^{\prime} \in W^{s}(q)$. Again, by the proximity of $f$ and $h_{a, b}$, and the fact that vectors with slope smaller than $1 / 10$ in $\tilde{R}_{3} \cap R$ are sent by $D h_{a, b}$ in vectors with slope smaller than $1 / 10$, and uniformly expanded, we have that the horizontal distance between $f(t)$ and $f\left(t^{\prime}\right)$ is uniformly expanded. Applying $f$ repeatedly, as long as the image is inside $\tilde{R}_{3} \cap R$, we have that the horizontal distance between the successive images of $t$ and $W^{s}(q)$ increases exponentially. Then the forward images of $t$ leave $R$ for some positive time.

Points of $R_{2}$ map to $R_{0}$ in backward time. Notice that $f^{-1}\left(R_{2}\right) \cap R \subset R_{0}$ since all the other regions in $R$ outside $\mathcal{D}$ are mapped forward to the region $\tilde{R}_{3}$, and so do not contain points of the backward image of $R_{2}$. Moreover, the unstable boundary of $R_{2}$ belongs to $W^{u}(p)$ and approaches $p$ as we apply $f^{-1}$, and the stable boundary cannot cross $W^{s}(q)$, then $f^{-1}\left(R_{2}\right)$ does not intersect $\mathcal{D}$. Since $f^{-1}\left(R_{2}\right) \cap R \subset R_{0}$, the points in there that are not in $W^{u}(p)$ leave $R$ for backward iterations. 


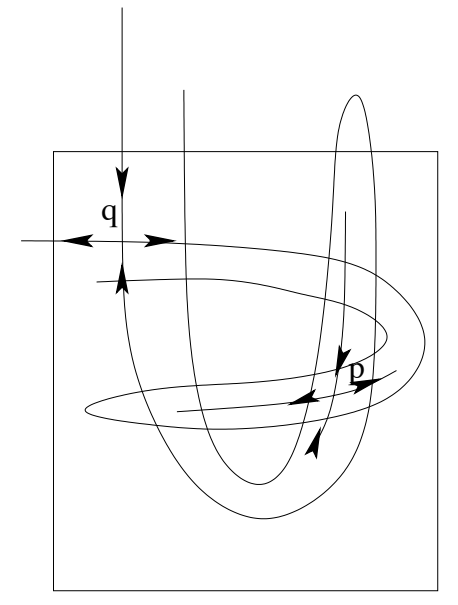

(a)

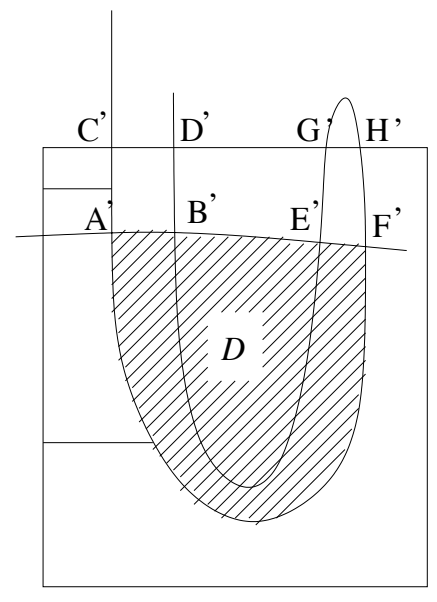

(b)

FiguRE 8. Invariant manifolds and the region $\mathcal{D}$ for $b<0$

Lemma 4. $\Omega \subset \overline{W^{u}(p)}$

Proof. Notice first of all that by the $\lambda$-lemma we have $q \in \overline{W^{u}(p)}$. Now suppose by contradiction that there exists $z=(x, y) \in \Omega$ with $z \notin \overline{W^{u}(p)}$. Then there exists $\varepsilon$ and an $\varepsilon$ neighbourhood $B_{\varepsilon}(z)$ of $z$ with $B_{\varepsilon}(z) \cap \overline{W^{u}(p)}=\emptyset$. Since $\Omega$ is $f$-invariant we have $f^{-n}(z) \in \Omega(f) \subset \mathcal{D}$ for all $n \in \mathbb{N}$ and therefore $z \in f^{n}(\mathcal{D})$ for all $n \in \mathbb{N}$. Notice that the boundary $\partial f^{n}(\mathcal{D}) \subset W^{u}(p) \cup f^{n}\left(E B^{s}\right)$ (where $E B^{s}$ denotes the piece of $W^{s}(q)$ between $E$ and $B$, as in Figure 6 . It is enough therefore to show that, for large $n$, the $\partial f^{n}(\mathcal{D})$ is $\varepsilon$-dense in $f^{n}(\mathcal{D})$ as this will imply that $B_{\varepsilon}(z) \cap \overline{W^{u}(p)} \neq \emptyset$, contradicting the assumptions. This follows easily from the fact that $f$ is (strongly) area contracting, and thus the area of $f^{n}(\mathcal{D})$ tends to zero as $n \rightarrow \infty$. In particular we must have that $B_{\varepsilon}(z) \cap \partial f^{n}(\mathcal{D}) \neq \emptyset$ for all $n \geq N$ sufficiently large. Moreover, the length of the part of the boundary which belongs to $W^{s}(q)$ also tends to zero. Thus most of the boundary belongs to $W^{u}(p)$ and thus we must have $B_{\varepsilon}(z) \cap W^{u}(p) \neq \emptyset$ for all $n$ sufficiently large.

\subsubsection{Completion of the proof.}

Proof of Proposition 1. Combining the results of the Lemmas stated above we have that $\Omega \subset \overline{W^{u}(p)} \cap \mathcal{D}$. The statement in the Proposition now follows immediately by observing that $\Omega \subset \mathcal{D}$ implies $\Omega \subset f(\mathcal{D})$ and that $f(\mathcal{D}) \subset[-2,2] \times[-4 b, 4 b]$ directly from the definition of $f$ if $\eta$ is sufficiently small.

Finally, in the case $b<0$, we consider the stable and unstable manifolds of $q$ crossing as in Figure 8 (the rectangle $R$ is exactly the same), determining the region $\mathcal{D}$ in this case. The proof is entirely analogous considering the points $A^{\prime}, B^{\prime}$, etc., corresponding to the points $A, B$, etc., above. 


\section{Hyperbolic COORDINATES AND CRITICAL POINTS}

The key idea of our whole strategy is the notion of dynamically defined critical point which relies on the fundamental notion of hyperbolic coordinates. In this section we introduce these notions and develop the main technical ideas which we will use. In Section 3.1 we clarify the relations between various constants used in the argument and introduce some preliminary geometric constructions. In Section 3.2 we discuss the definition and basic theory of hyperbolic coordinates. In Section 3.3 we introduce the idea of admissible curves and prove certain estimates concerning the images of admissible curves. Finally, in Section 3.4 we introduce the notion of dynamically defined critical point and prove that such critical points always exist in images of certain admissible curves.

\subsection{Preliminary geometric definitions and fixing the constants.}

3.1.1. Fixing the constants. We now explain the required relations between the different constants used in the proof, and the order in which these constants are chosen. All constants are positive. First of all we fix two constants

$$
\delta=1 / 10 \quad \text { and } \alpha=1 / 2 .
$$

These will be introduced in Sections 3.1.2 and 3.3 below. Even though we specify the actual numerical value of these constants we shall continue to use the constants in the argument because they have some specific geometric meaning and it is useful to keep track of their occurrence throughout the paper. We then fix a constant $k_{0}$ large enough so that

$$
\frac{\sqrt{\delta}}{2 \sqrt{3}}(\sqrt{3 / \sqrt{5}})^{k_{0}-1}>1
$$

In Section 3.1.3 we fix a constant $\varepsilon$ which will then remain unchanged. Finally, at some finite number of places in the argument, we will require $a$ to be sufficiently close to 2 and $|b|$ and $\eta$ to be sufficiently small.

We remark that we can suppose that $a$ is close to 2 without compromising the fact that hyperbolicity holds for all larger values of $a$. Indeed, once we fix a neighbourhood of 2 in the $a$ parameter space, we can always guarantee uniform hyperbolicity for values of $a>2$ outside this neighbourhood by taking $|b|$ and $\eta$ sufficiently small, (depending on this neighbourhood).

3.1.2. The fixed point neighbourhoods. Recall first of all that the map $f_{*}=h_{2,0}$ has two fixed points $p_{*}$ and $q_{*}=(-1,0)$ with $f_{*}(1,0)=q_{*}$. For $\delta=1 / 10$ we let $\mathcal{Q}=\mathcal{Q}_{0}:=B_{\delta}\left(q_{*}\right)$ be the open ball of radius $\delta$ centred at $q_{*}$ and $\mathcal{V}=\mathcal{V}_{0}$ be the component of $f_{*}^{-1}(\mathcal{Q})$ not intersecting $\mathcal{Q}$, see Figure 9 . The expanding eigenvalue at the point $q_{*}$ is equal to 4 and so we can suppose that $|a-2|,|b|, \eta$, are all small enough so that $\left\|D f_{z}\right\|>3$ for all $z \in \mathcal{Q}$. Then, for $n \geq 0$, let

$$
\mathcal{Q}_{n}(f)=\bigcap_{i=0}^{n} f^{-i}\left(\mathcal{Q}_{0}\right) \quad \text { and } \quad \mathcal{V}_{n}(f)=f^{-1}\left(\mathcal{Q}_{n}(f)\right) \cap \mathcal{V}
$$

Notice that $\mathcal{V}_{n}$ is just the component of $f^{-1}\left(\mathcal{Q}_{n}(f)\right)$ containing $(1,0)$. Since $\mathcal{Q}_{n}$ is a 


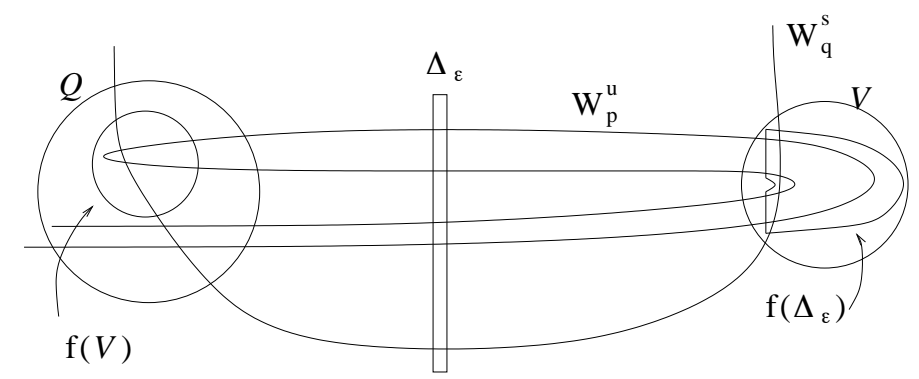

Figure 9. The neighbourhoods $\mathcal{Q}$ and $\mathcal{V}$

neighbourhood of $q$ for every $n, \mathcal{V}_{n} \backslash f^{-1}\left(W_{\delta}^{s}(q)\right)$, where $W_{\delta}^{s}(q)$ denotes the connected component containing $q$ of $W^{s}(q) \cap \mathcal{Q}_{0}$, has two components: we let

$$
\mathcal{V}_{n}^{-}=V_{n} \cap \mathcal{D} \quad \text { and } \quad \mathcal{V}_{n}^{+}=\mathcal{V}_{n} \backslash \mathcal{V}_{n}^{-}
$$

Notice that a piece of $W^{s}(q)$ forms the boundary between $\mathcal{V}_{n}^{+}$and $\mathcal{V}_{n}^{-}$. We mention for future reference a simple estimate which we shall use below.

Lemma 5. $d\left(z, f^{-1}\left(W_{\delta}^{s}(q)\right)\right) \geq \delta / 5^{k}$ for all $z \in \mathcal{V}_{k} \backslash \mathcal{V}_{k+1}$.

Proof. $z \in \mathcal{V}_{k} \backslash \mathcal{V}_{k+1}$ implies, by definition, $d\left(z_{k+1}, q\right) \geq \delta$. For points $z$ close to $f^{-1}\left(W_{\delta}^{s}(q)\right)$ this also means $d\left(z_{k+1}, W_{\delta}^{s}(q)\right) \geq \delta$ since such points come very close to the fixed point $q$ and escape the $\delta$ neighbourhood of $q$ along the direction of $W^{u}(q)$. Thus, using the fact that the norm of the derivative $D f$ in $\mathcal{D}$ is uniformly bounded above by 5 we obtain the result.

3.1.3. The critical neighbourhood. For $\varepsilon>0$ we define a critical neighbourhood

$$
\Delta_{\varepsilon}=(-\varepsilon, \varepsilon) \times(-4 b, 4 b) .
$$

Notice that we can take $\varepsilon$ sufficiently small so that $q_{f} \in f(\mathcal{V})$ and

$$
f\left(\Delta_{\varepsilon}\right) \subset \mathcal{V}_{k_{0}} .
$$

From now on we consider $\varepsilon$ fixed. We also let

$$
\Delta=\Delta_{a}=\left\{x \in \Delta_{\varepsilon}: f(x) \notin \mathcal{D}\right\} .
$$

For $a$ sufficiently close to 2 and $|b|$ and $\eta$ sufficiently small we have uniform hyperbolicity outside $\Delta_{\varepsilon}$. We state this fact more formally in the following

Lemma 6. For every $\hat{\lambda} \in(0, \log 2)$ and $|a-2|,|b|, \eta>0$ sufficiently small, there exists $a$ constant $C_{\varepsilon}>0$ such that for all $k \geq 1$ and points $z$ with $z, f(z), \ldots, f^{k-1}(z) \notin \Delta_{\varepsilon}$, and vector $v$ with slope $<\alpha$ we have

$$
\begin{gathered}
\text { slope } D f_{z}^{k}(v)<\alpha, \\
\left\|D f_{z}^{k}(v)\right\| \geq C_{\varepsilon} e^{\hat{\lambda} k}\|v\| .
\end{gathered}
$$


If, moreover, $f^{k}(z) \in \Delta$ then we have

$$
\left\|D f_{z}^{k}(v)\right\| \geq e^{\hat{\lambda} k}\|v\|
$$

Proof. This is a standard result (see for example [BC91] or [MV93]) and so we omit the details. We just mention that it follows from the fact that the limiting one-dimensional map $h_{2,0}$ satisfies uniform expansivity estimates outside an arbitrary critical neighbourhood $\Delta_{\varepsilon}$ (with constant $\hat{\lambda}$ arbitrarily close to $\log 2$ but constant $C_{\varepsilon}$ depending on $\varepsilon$ and arbitrarily small for $\varepsilon$ small), see e.g. [dMvS93].

Considering this one-dimensional map as embedded in the space of two-dimensional maps and using the fact that uniform hyperbolicity is an open condition we obtain the statement in the Lemma for $|b|, \eta \neq 0$ sufficiently small.

3.2. Hyperbolic coordinates. The notion of Hyperbolic Coordinates is inspired by some constructions in [BC91, MV93], developed in [LV03] and formalized in [HL06] as an alternative framework with which to approach the classical theory of invariant manifolds. Here we review the basic definitions and theory to the extent to which they will be required for our purposes.

3.2.1. Hyperbolicity of compositions of linear maps. We recall the notion of hyperbolic coordinates and give the basic definitions and properties in the general context of $C^{2}$ diffeomorphisms of a Riemannian surface $M$. For $z \in M, k \geq 1$ we let

$$
F_{k}(z)=\left\|D f_{z}^{k}\right\| \quad \text { and } \quad E_{k}(z)=\left\|\left(D f_{z}^{k}\right)^{-1}\right\|^{-1}
$$

denote the maximum expansion and the maximum contraction respectively of $D f_{z}^{k}$. Then we think of the quantity

$$
H_{k}(z)=E_{k}(z) / F_{k}(z)
$$

as the hyperbolicity of $D f_{z}^{k}$. Notice that $H_{k} \leq 1$ always. The condition $H_{k}=E_{k} / F_{k}<1$ implies that the linear map $D f^{k}$ maps the unit circle $\mathcal{S} \subset T_{z} M$ to an ellipse $\mathcal{S}_{k}=D f_{z}^{k}(\mathcal{S}) \subset$ $T_{f^{k}(z)} M$ with well defined major and minor axes. The unit vectors $e^{(k)}, f^{(k)}$ which are mapped to the minor and major axis respectively of the ellipse, and are thus the most contracted and most expanded vectors respectively, are given analytically as solutions to the differential equation $d\left\|D f_{z}^{k}(\cos \theta, \sin \theta)\right\| / d \theta=0$ which can be solved to give the explicit formula

$$
\tan 2 \theta=\frac{2\left[\left(\partial_{x} f_{1}^{k}\right)\left(\partial_{y} f_{1}^{k}\right)+\left(\partial_{x} f_{2}^{k}\right)\left(\partial_{y} f_{2}^{k}\right)\right]}{\left(\partial_{x} f_{1}^{k}\right)^{2}+\left(\partial_{x} f_{2}^{k}\right)^{2}-\left(\partial_{y} f_{1}^{k}\right)^{2}-\left(\partial_{y} f_{2}^{k}\right)^{2}}
$$

Here $f=\left(f_{1}, f_{2}\right)$ are the two coordinate functions of $f$. Notice that $e^{(k)}$ and $f^{(k)}$ are always orthogonal and do not in general correspond to the stable and unstable eigenspaces of $D f^{k}$. 
3.2.2. Hyperbolic coordinates and stable and unstable foliations. We define the hyperbolic coordinates of order $k$ at the point $z$ as the orthogonal coordinates $\mathcal{H}_{k}(z)$ given at $z$ by the most contracted and most expanded directions for $D f_{z}^{k}$. If $f$ is $C^{2}$ and $H_{k}(z)<$ 1 then hyperbolic coordinates are defined in some neighbourhood of $z$ and define two orthogonal $C^{1}$ vector fields. In particular they are locally integrable and thus give rise to two orthogonal foliations. We let $\mathcal{E}^{(k)}$ denote the stable foliation of order $k$ formed by the integral curves of the vector field $\left\{e^{(k)}\right\}$ and $\mathcal{F}^{(k)}$ denote the unstable foliation of order $k$ formed by the integral curves of the vector field $\left\{f^{(k)}\right\}$.

3.2.3. Hyperbolic coordinates for Hénon-like maps. A crucial property of hyperbolic coordinates and finite order stable and unstable foliations is that, under very mild assumptions, they converge in quite a strong sense as $k \rightarrow \infty$. We formulate a version of this property here in our specific context.

Proposition 2. For every $k \geq 1$, hyperbolic coordinates $\mathcal{H}_{k}$ and stable and unstable foliations $\mathcal{E}^{(k)}$ and $\mathcal{F}^{(k)}$ are defined in $\mathcal{V}^{+} \cup \mathcal{V}_{k}^{-}$Moreover

(1) the angle between each stable direction $e^{(k)}$ and the slope of $f^{-1}\left(W_{\delta}^{s}(q)\right)(\approx 2)$.

(2) the curvature of each stable leaf,

are both $\lesssim b$. Also, the $C^{2}$ distance between leafs of $\mathcal{E}^{(k)}$ and leaves $\mathcal{E}^{(k+1)}$ is $\lesssim b k$.

Proof. Analogous convergence results are formulated and proved in great generality in [HL06] under weak (subexponential) growth of the derivative. Here we shall need only some very particular cases of these estimates and therefore we first describe the specific setting in which they will be applied here. The main ingredient for the proof is that fact that by our choice of $\delta$ and assuming that $|a-2|,|b|$ and $\eta$ are small enough we have that $\left\|D f(z)-D f_{*}\left(q_{*}\right)\right\|$ is small for all $z \in \mathcal{Q}$ and thus in particular

$$
E_{k}\left(z_{0}\right) \leq b^{k} \quad \text { and } \quad F_{k}\left(z_{0}\right) \geq 3^{k} \quad \forall z_{0} \in \mathcal{V}_{k}
$$

It follows immediately that hyperbolic coordinates, and their associated foliations, of order $k$ are well defined in $\mathcal{V}_{k}$. Points in $\mathcal{V}_{k}^{-}$are then re-injected into $\mathcal{D} \backslash \mathcal{Q}$ and these hyperbolicity estimates can no longer be guaranteed, a priori, for all time. Points in $\mathcal{V}_{k}^{+}$however are outside $\mathcal{D}$ and therefore, by the arguments of Section 2, eventually escape towards infinity. In particular the required hyperbolicity conditions can be guaranteed to hold for all positive iterates. This implies that hyperbolic coordinates of order $k$ are well defined in $\mathcal{V}^{+} \cup \mathcal{V}_{k}^{-}$ as in the statement of the proposition.

The statements about the direction of the stable directions, the curvature of the leaves and the $C^{2}$ distance between stable leaves of different orders, all follow directly from [HL06, Main Theorem]. These calculations are purely technical and do not add to our geometrical understanding of this situation, we therefore omit the details and refer the reader to that paper. 
3.3. Admissible curves. Recall that the curvature $\kappa(s)$ of a parametrized curve $\gamma(s)=$ $(x(s), y(s))$ is given by

$$
\kappa(s)=\frac{|\dot{x} \ddot{y}-\dot{y} \ddot{x}|}{\|(\dot{x}, \dot{y})\|^{3}}=\frac{|\dot{\gamma} \times \ddot{\gamma}|}{|\dot{\gamma}|^{3}} .
$$

The equivalence between the two formulas is given by the formula $\left(v_{1}, v_{2}\right) \times\left(w_{1}, w_{2}\right)=$ $v_{1} w_{2}-v_{2} w_{1}$.

Definition 1. For $\alpha>0$, we say that a $C^{2}$ curve $\gamma=\gamma(s)=(x(s), y(s))$ is admissible if $|\dot{y}(s)| /|\dot{x}(s)|<\alpha$ and $|\kappa(s)|<\alpha$ for all $s$.

We remark that both the curvature and the slope of tangent vectors of a curve are independent of the parametrization, and thus so is the definition of admissibility. We shall want to compare the curvature at a point of a curve and at the corresponding point of its image. So, we suppose $\gamma_{i-1}(s)$ is a parametrized $C^{2}$ curve and $\gamma_{i}(s)=f\left(\gamma_{i-1}(s)\right)$. For simplicity we shall often omit the parameter $s$ and simply write $D f$ to denote the derivative at the point $\gamma_{i-1}(s)$.

Proposition 3. Let $\left\{\gamma_{i}\right\}_{i=0}^{n}$ be a sequence of $C^{2}$ curves with $\gamma_{i}=f^{i}\left(\gamma_{0}\right)$. Suppose that for some $s, n$ is a "hyperbolic time" in the sense that

$$
\left\|\dot{\gamma}_{n}(s)\right\| \geq C e^{\lambda j}\left\|\dot{\gamma}_{n-j}(s)\right\|
$$

for all $j=0, . ., n-1$. Then for $|b|, \eta$ sufficiently small, $\kappa_{0}(s)<\alpha$ implies $\kappa_{n}(s)<\kappa_{0}(s)<\alpha$.

Corollary 1. If $\gamma \subset \mathcal{D} \backslash \Delta_{\varepsilon}$ is admissible, then $f(\gamma)$ is also admissible

Proof. This follows from Proposition 3 and the hyperbolicity outside $\Delta_{\varepsilon}$. Condition (5) implies that the slope of each tangent vector to $f(\gamma)$ is $<\alpha$ and condition (6) together with Lemma 3 gives the curvature $<\alpha$.

To prove Proposition 3 we first prove a general curvature estimate. We fix some bounded neighbourhood $\hat{R}$ of $R$ and, as above, we suppose $\left\{\gamma_{i}\right\}_{i=0}^{n}$ is a sequence of $C^{2}$ (not necessarily admissible) curves with $\gamma_{i}=f^{i}\left(\gamma_{0}\right)$, all contained in $\hat{R}$.

Lemma 7. There exists $K>0$ independent of $a, b, \eta$ such that for all $i=1, \ldots, n$ we have

$$
\kappa_{i}(s) \leq K(b+\eta) \frac{\left|\dot{\gamma}_{i-1}(s)\right|^{3}}{\left|\dot{\gamma}_{i}(s)\right|^{3}} \kappa_{i-1}(s)+K(b+\eta) \frac{\left|\dot{\gamma}_{i-1}(s)\right|^{3}}{\left|\dot{\gamma}_{i}(s)\right|^{3}}
$$

Proof. We use the formula $\kappa=|\dot{\gamma} \times \ddot{\gamma}| /|\dot{\gamma}|^{3}$ for the curvature. We have

$$
\dot{\gamma}_{i}=(D f) \dot{\gamma}_{i-1}=\left(\begin{array}{ll}
f_{1, x} & f_{1, y} \\
f_{2, x} & f_{2, y}
\end{array}\right) \dot{\gamma}_{i-1}=\left(\begin{array}{cc}
-2 a x_{i-1}+\varphi_{1, x} & 1+\varphi_{1, y} \\
b+\varphi_{2, x} & \varphi_{2, y}
\end{array}\right) \dot{\gamma}_{i-1}
$$

and

$$
\ddot{\gamma}_{i}=\left(\begin{array}{cc}
\nabla f_{1, x} \cdot \dot{\gamma}_{i-1} & \nabla f_{1, y} \cdot \dot{\gamma}_{i-1} \\
\nabla f_{2, x} \cdot \dot{\gamma}_{i-1} & \nabla f_{2, y} \cdot \dot{\gamma}_{i-1}
\end{array}\right) \dot{\gamma}_{i-1}+(D f) \ddot{\gamma}_{i-1}
$$

Therefore $\dot{\gamma}_{i} \times \ddot{\gamma}_{i}$ is given by

$$
(D f) \dot{\gamma}_{i-1} \times\left(\begin{array}{cc}
\nabla f_{1, x} \cdot \dot{\gamma}_{i-1} & \nabla f_{1, y} \cdot \dot{\gamma}_{i-1} \\
\nabla f_{2, x} \cdot \dot{\gamma}_{i-1} & \nabla f_{2, y} \cdot \dot{\gamma}_{i-1}
\end{array}\right) \dot{\gamma}_{i-1}+(D f) \dot{\gamma}_{i-1} \times(D f) \ddot{\gamma}_{i-1}
$$


where

$$
\nabla f_{1, x}=\left(\begin{array}{c}
-2 a+\varphi_{1, x x} \\
\varphi_{1, x y}
\end{array}\right)
$$

and

$$
\nabla f_{1, y}=\left(\begin{array}{c}
\varphi_{1, x y} \\
\varphi_{1, y y}
\end{array}\right) ; \nabla f_{2, x}=\left(\begin{array}{c}
\varphi_{2, x x} \\
\varphi_{2, x y}
\end{array}\right) ; \nabla f_{2, y}=\left(\begin{array}{c}
\varphi_{2, x y} \\
\varphi_{2, y y}
\end{array}\right)
$$

We shall estimate the two terms of (10) separately. These will yield the two terms in the statement of the Proposition. For the second term we have

$$
\left|(D f) \dot{\gamma}_{i-1} \times(D f) \ddot{\gamma}_{i-1}\right|=|\operatorname{Det}(D f)|\left|\dot{\gamma}_{i-1} \times \ddot{\gamma}_{i-1}\right|=|\operatorname{Det}(D f)| \kappa_{i-1}\left|\gamma_{i-1}\right|^{3} .
$$

Indeed, for the first equality, $\left|\dot{\gamma}_{i-1} \times \ddot{\gamma}_{i-1}\right|$ is the area of the parallelogram defined by the two vectors $\dot{\gamma}_{i-1}$ and $\ddot{\gamma}_{i-1}$, and $\left|(D f) \dot{\gamma}_{i-1} \times(D f) \ddot{\gamma}_{i-1}\right|$ is the are of the parallelogram defined by the two vectors $\dot{\gamma}_{i-1}$ and $\ddot{\gamma}_{i-1}$ which of course just the image of the first parallelogram under $D f$. The second equality just follows immediately from the definition of $\kappa_{i-1}$. So it just remains to show that the value of $|\operatorname{Det}(D f)|$ is bounded above by some multiple of $b$ and $\eta$. Indeed, writing $f=h+\varphi$ we have, by the "row-linearity" of the determinant,

$$
\begin{aligned}
\operatorname{Det}(D f) & =\operatorname{Det}\left(\begin{array}{ll}
h_{1 x}+\varphi_{1 x} & h_{1 y}+\varphi_{1 y} \\
h_{2 x}+\varphi_{2 x} & h_{2 x}+\varphi_{2 y}
\end{array}\right) \\
& =\operatorname{Det}\left(\begin{array}{cc}
h_{1 x} & h_{1 y} \\
h_{2 x}+\varphi_{2 x} & h_{2 x}+\varphi_{2 y}
\end{array}\right)+\operatorname{Det}\left(\begin{array}{cc}
\varphi_{1 x} & \varphi_{1 y} \\
h_{2 x}+\varphi_{2 x} & h_{2 x}+\varphi_{2 y}
\end{array}\right) \\
& =\operatorname{Det}\left(\begin{array}{ll}
h_{1 x} & h_{1 y} \\
h_{2 x} & h_{2 x}
\end{array}\right)+\operatorname{Det}\left(\begin{array}{ll}
h_{1 x} & h_{1 y} \\
\varphi_{2 x} & \varphi_{2 y}
\end{array}\right)+\operatorname{Det}\left(\begin{array}{ll}
\varphi_{1 x} & \varphi_{1 y} \\
h_{2 x} & h_{2 x}
\end{array}\right)+\operatorname{Det}\left(\begin{array}{ll}
\varphi_{1 x} & \varphi_{1 y} \\
\varphi_{2 x} & \varphi_{2 y}
\end{array}\right)
\end{aligned}
$$

Using $h_{1 x}=-2 a, h_{1 y}=1, h_{2 x}=b, h_{2 y}=0$ and $\|\varphi\|_{C^{2}} \leq \eta$ this gives

$$
\operatorname{Det}(D f) \leq b+(2 a \eta+\eta)+(2 a \eta+\eta)+\eta=b+4 a \eta+3 \eta \leq b+12 \eta
$$

where in the last step we have used the fact that $a$ is close to 2. Substituting this above gives the required bound for the second term of (10). To bound the first term we write

$$
(D f) \dot{\gamma}_{i-1} \times\left(\begin{array}{cc}
\nabla f_{1, x} \cdot \dot{\gamma}_{i-1} & \nabla f_{1, y} \cdot \dot{\gamma}_{i-1} \\
\nabla f_{2, x} \cdot \dot{\gamma}_{i-1} & \nabla f_{2, y} \cdot \dot{\gamma}_{i-1}
\end{array}\right) \dot{\gamma}_{i-1}=\left(\begin{array}{ll}
a_{1} & b_{1} \\
c_{1} & d_{1}
\end{array}\right)\left(\begin{array}{l}
v_{1} \\
v_{2}
\end{array}\right) \times\left(\begin{array}{ll}
a_{2} & b_{2} \\
c_{2} & d_{2}
\end{array}\right)\left(\begin{array}{l}
v_{1} \\
v_{2}
\end{array}\right)
$$

Then the norm of this expression is bounded above by

$$
\begin{aligned}
& \left|a_{1} c_{1} v_{1}^{2}+a_{1} d_{2} v_{1} v_{2}+b_{1} c_{2} v_{1} v_{2}+b_{1} d_{2} v_{2}^{2}-a_{2} c_{1} v_{1}^{2}-a_{2} d_{1} v_{1} v_{2}-b_{2} c_{1} v_{1} v_{2}-d_{1} b_{2} v_{2}^{2}\right| \\
\leq & \max \left\{\left|a_{1} c_{2}-a_{2} c_{1}\right|,\left|b_{1} d_{2}-d_{1} b_{2}\right|+\left|a_{1} d_{2}-c_{1} b_{2}\right|+\left|b_{1} c_{2}-a_{2} d_{1}\right|\right\}\left(v_{1}^{2}+v_{2}^{2}\right) \\
\leq & 4 \max \left\{\left|a_{1} c_{2}-a_{2} c_{1}\right|,\left|b_{1} d_{2}-d_{1} b_{2}\right|,\left|a_{1} d_{2}-c_{1} b_{2}\right|,\left|b_{1} c_{2}-a_{2} d_{1}\right|\right\}\left(v_{1}^{2}+v_{2}^{2}\right) \\
\leq & 8 \max \left\{\left|a_{1} c_{2}\right|,\left|a_{2} c_{1}\right|,\left|b_{1} d_{2}\right|,\left|d_{1} b_{2}\right|,\left|a_{1} d_{2}\right|,\left|c_{1} b_{2}\right|,\left|b_{1} c_{2}\right|,\left|a_{2} d_{1}\right|\right\}\left|\dot{\gamma}_{i-1}\right|^{2} .
\end{aligned}
$$

All the terms contain a factor $\dot{\gamma}_{i-1}$; each of the terms $b_{2}, c_{2}, d_{2}$, see $(12)$, contains a bounded constant multiplied by the factor $\eta$; the term $a_{2}$, see (11), is of the order of $2 a$ but here it 
is multiplied by either $c_{1}$ or $d_{1}$ each one of which contains a term which is bounded by $\eta$. Therefore, there exists a constant $K>0$ such that

$$
\left|(D f) \dot{\gamma}_{i-1} \times\left(\begin{array}{cc}
\nabla f_{1, x} \cdot \dot{\gamma}_{i-1} & \nabla f_{1, y} \cdot \dot{\gamma}_{i-1} \\
\nabla f_{2, x} \cdot \dot{\gamma}_{i-1} & \nabla f_{2, y} \cdot \dot{\gamma}_{i-1}
\end{array}\right) \dot{\gamma}_{i-1}\right| \leq K \eta\left|\gamma_{i-1}\right|^{3}
$$

Proof of Proposition 3. Applying Lemma 7 recursively we get

$$
\begin{aligned}
\kappa_{n}(s) & \leq K(b+\eta) \kappa_{n-1}(s) \frac{\left|\dot{\gamma}_{n-1}\right|^{3}}{\left|\dot{\gamma}_{n}\right|^{3}}+K(b+\eta) \frac{\left|\dot{\gamma}_{n-1}\right|^{3}}{\left|\dot{\gamma}_{n}\right|^{3}} \\
& \leq(K(b+\eta))^{2} \kappa_{n-2} \frac{\left|\dot{\gamma}_{n-2}\right|^{3}}{\left|\dot{\gamma}_{n}\right|^{3}}+(K(b+\eta))^{2} \frac{\left|\dot{\gamma}_{n-2}\right|^{3}}{\left|\dot{\gamma}_{n}\right|^{3}}+K(b+\eta) \frac{\left|\dot{\gamma}_{n-1}\right|^{3}}{\left|\dot{\gamma}_{n}\right|^{3}} \\
& \leq \ldots
\end{aligned}
$$

Using the expansivity assumption and $b, \eta$ small, this gives

$$
\kappa_{n}(s) \leq \frac{1}{C^{3}}\left(K(b+\eta) e^{-\lambda}\right)^{n} \kappa_{0}(s)+\frac{1}{C^{3}} \frac{K(b+\eta) e^{-\lambda}}{1-K(b+\eta) e^{-\lambda}} \leq \kappa_{0}(s) \leq \alpha .
$$

3.4. Critical points. The next Proposition makes precise the notion of a critical point of order $k$. We recall that $\gamma$ is a $C^{2}$ admissible curve if all its tangent vectors have slope $<\alpha$ and it has curvature $<\alpha$. We say that $\gamma$ is a long admissible curve if it is an admissible curve which crosses the entire length of $\Delta_{\varepsilon}$.

Proposition 4. Let $\gamma \subset \Delta_{\varepsilon} \cap \mathcal{D}$ be a long admissible curve. Then there exists a unique point $c^{(k)} \in \gamma$ such that $\gamma_{0}=f(\gamma)$ has a (quadratic) tangency at $c_{0}^{(k)}=f\left(c^{(k)}\right) \in \mathcal{V}_{k}^{-} \cup \mathcal{V}^{+}$with the stable foliation $\mathcal{E}^{(k)}$, for any $k \geq k_{0}$. Moreover there exists a constant $K$, independent of $b, \eta$, such that $d\left(c_{0}^{(k)}, c_{0}^{(k+1)}\right) \leq K b^{k}$. In particular, the sequence $\left\{c_{0}^{(k)}\right\}$ is Cauchy.

Definition 2. We call $c^{(k)}$ and $c_{0}^{(k)}$ respectively a critical point and critical value of order $k$, associated to the long admissible curve $\gamma$.

We remark that critical values $c_{0}^{(k)}$ of finite order are not guaranteed to be outside $\mathcal{D}$, however we shall show below that their limit points as $k \rightarrow \infty$, i.e. the "real" critical points always fall strictly outside $\mathcal{D}$ for $a>a^{*}$.

Given a parametrized curve $\gamma_{0}=\gamma_{0}(t)$ and its image $\gamma_{1}=\gamma_{1}(t)=f\left(\gamma_{0}(t)\right)$ we denote by $\kappa_{0}(t)$ the curvature of $\gamma_{0}$ at the point $\gamma_{0}(t)$ and by $\kappa_{1}(t)$ the curvature of $\gamma_{1}$ at the point $\gamma_{1}(t)$.

Lemma 8. Let $\gamma_{0}(t)$ be an admissible curve and let $\gamma_{1}(t)=f(\gamma(t))=\left(\xi_{1}(t), \eta_{1}(t)\right)$. Suppose that for some $t$ we have $\dot{\eta}_{1}(t) \neq 0$ and $\left|\dot{\xi}_{1}(t) / \dot{\eta}_{1}(t)\right|<1$. Then $\left|\kappa_{1}(t)\right|>a / b \gg 1$.

Lemma 8 essentially says that if the tangent direction of the image of an admissible curve at a certain point is roughly vertical (or at least contained in the "vertical" cone between the positive and the negative diagonals) then the curvature at this point is strictly bounded 
away from 0 . This does not apply to admissible curves outside $\Delta_{\varepsilon}$ since we have shown above ( Corollary 1) that images of such curves are still admissible and therefore their tangent directions are roughly horizontal. We will instead apply it below to the images of admissible curves inside $\Delta_{\varepsilon}$ as a way of pinpointing the location of folds.

Proof. First recall that the curvature $\kappa_{1}(t)$ is independent of the choice of parametrization and also the condition $\left|\dot{\xi}_{1}(t) / \dot{\eta}_{1}(t)\right|<1$ is independent of the parametrization since $\left|\dot{\xi}_{1}(t) / \dot{\eta}_{1}(t)\right|$ is just the slope of the tangent vector. Therefore we choose the parametrization

$$
\gamma_{0}(t)=(t, y(t))
$$

For simplicity we also omit the subscript 1 from the coordinate functions of $\gamma_{1}$ and just write $\gamma_{1}(t)=(\xi(t), \eta(t))$. From the definition of $f$ we have

$$
\begin{aligned}
& (\xi(t), \eta(t))=\left(1+a t^{2}+y(t)+\varphi_{1}\left(\gamma_{0}(t)\right), b t+\varphi_{2}\left(\gamma_{0}(t)\right)\right) \\
& (\dot{\xi}(t), \dot{\eta}(t))=\left(-2 a t+\dot{y}(t)+\nabla \varphi_{1}\left(\gamma_{0}(t)\right) \cdot \dot{\gamma}_{0}(t), b+\nabla \varphi_{2}\left(\gamma_{0}(t)\right) \cdot \dot{\gamma}_{0}(t)\right) \\
& (\ddot{\xi}(t), \ddot{\eta}(t))=\left(-2 a+\dot{y}(t)+D^{2} \varphi_{1}\left(\gamma_{0}(t)\right)\left[\dot{\gamma}_{0}(t)\right]^{2}, D^{2} \varphi_{2}\left(\gamma_{0}(t)\right)\left[\dot{\gamma}_{0}(t)\right]^{2}\right)
\end{aligned}
$$

Choosing $\eta$ sufficiently small, for example so that $4\left\|\nabla \varphi_{2}\left(\gamma_{0}(t)\right)\right\|(1+\alpha)<b$ this implies

$$
3 b / 4 \leq|\dot{\eta}(t)| \leq 5 b / 4 \text {. }
$$

We can now compute the curvature $\kappa_{1}(t)$. First notice that the condition $\left|\dot{\xi}_{1}(t) / \dot{\eta}_{1}(t)\right|<1$ implies in particular $\|(\dot{\xi}(t), \dot{\eta}(t))\| \leq \sqrt{2}|\dot{\eta}(t)|$. Then we have

$$
\kappa_{1}(t)=\frac{|\ddot{\xi}(t) \dot{\eta}(t)-\dot{\xi}(t) \ddot{\eta}(t)|}{\|(\dot{\xi}(t), \dot{(} \eta(t))\|^{3}} \geq \frac{|\ddot{\xi}(t) \dot{\eta}(t)-\dot{\xi}(t) \ddot{\eta}(t)|}{4|(\dot{\eta}(t))|^{3}}
$$

Dividing numerator and denominator by $|\dot{\eta}(t)|$, using the condition $\left|\dot{\xi}_{1}(t) / \dot{\eta}_{1}(t)\right|<1$ and (13) we get

$$
\kappa_{1}(t) \geq \frac{\left|\ddot{\xi}(t)-\frac{\dot{\xi}(t)}{\dot{\eta}(t)} \ddot{\eta}(t)\right|}{4|(\dot{\eta}(t))|^{2}} \geq \frac{|\ddot{\xi}(t)|-\left|\frac{\dot{\xi}(t)}{\dot{\eta}(t)}\right||\ddot{\eta}(t)|}{4|(\dot{\eta}(t))|^{2}} \geq \frac{|\ddot{\xi}(t)|-|\ddot{\eta}(t)|}{4|(\dot{\eta}(t))|^{2}} \geq \frac{|\ddot{\xi}(t)|-|\ddot{\eta}(t)|}{7 b^{2}}
$$

Finally, from the formulas for $\ddot{\xi}(t)$ and $\ddot{\eta}(t)$ and the fact that $|\dot{y}(t)| \leq \alpha$ by the admissibility of $\gamma_{0}$, we get

$$
|\ddot{\xi}(t)|-|\ddot{\eta}(t)| \geq 2 a-\alpha-2\|\varphi\|_{C^{2}} \geq a
$$

as long as $\eta$ is sufficiently small.

Proof of Proposition 4. The existence of a tangency between $f(\gamma)$ and the stable foliation $\mathcal{E}^{(k)}$ follows by the simple geometric observation that the image of a long admissible curve necessarily "changes direction" between one endpoint and the other. Thus, by a simple Intermediate Value argument it follows that there is some point of tangency.

Now, Proposition 2 says that the leaves of the stable foliations $\mathcal{E}^{(k)}$ are close to the piece of stable manifold $f^{-1}\left(W_{\delta}^{s}(q)\right.$ and thus have slope close to 2, and that their curvature is small. In particular the point of tangency must occur at some point at which the tangent 

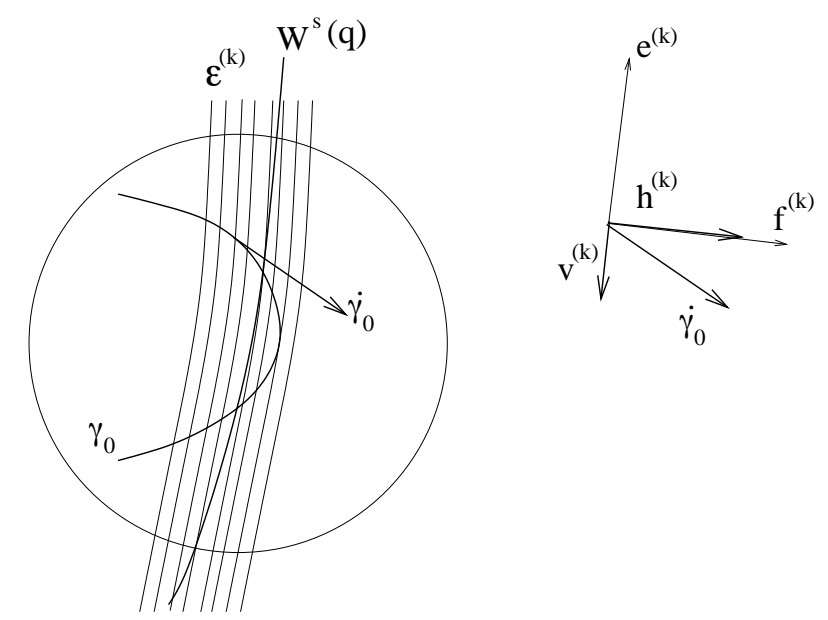

FiguRE 10. Hyperbolic coordinates

direction to $f(\gamma)$ is close to 2 and therefore Proposition 4 shows that at this point of tangency $f(\gamma)$ has strictly positive curvature. This implies that this tangency is quadratic as well as unique.

\section{Hyperbolicity estimates}

This is the final and main section of the paper. We apply the notion of hyperbolic coordinates and dynamical defined critical points to prove Theorem 1. In section 4.1 we combine the hyperbolic coordinates and the curvature estimates to show that all components of the unstable manifold $W^{u}(p)$ in $\Delta_{\varepsilon}$ are almost horizontal curves with small curvature. In particular they all have well-defined critical points. In section 4.2 we take advantage of the structure of critical points on such components to show that points in the critical region $\Delta_{\varepsilon} \backslash \Delta$ recover hyperbolicity after some bounded number of iterations depending only on the parameter $a$. In Section 4.3 we then extend these estimates to uniform expansion estimates on all of $W^{u}(p)$ with a hyperbolicity constant $C_{a}$ depending only on the parameter. In Section 4.4 we then show how to extend this hyperbolicity to the closure of $W^{u}(p)$ and thus to the whole nonwandering set $\Omega$. Finally, in Section 4.5, we consider the bifurcation parameter value $a=a^{*}$ and show that all Lyapunov exponents are uniformly bounded away from 0 .

\subsection{Shadowing. Let}

$$
\lambda=\min \left\{\frac{1}{2} \ln \frac{3}{\sqrt{5}}, \hat{\lambda}\right\} .
$$

Proposition 5. For all $a \geq a^{*}$ all components of $W^{u}(p) \cap \Delta_{\varepsilon}$ are long admissible curves. Moreover, for all $z \in W^{u}(p) \cap\left(\Delta_{\varepsilon} \backslash \Delta\right)$ and any vector $v$ tangent to $W^{u}(p)$ at $z$ and $k \geq 1$ 
such that $f(z) \in \mathcal{V}_{k} \backslash \mathcal{V}_{k+1}$ we have

$$
\left\|D f_{z}^{k}(v)\right\| \geq e^{\lambda k}\|v\|
$$

We emphasize that Proposition 5 holds also for parameter values for which the first tangency occurs.

Proof. We first prove the expansivity statement and then the admissibility of leaves of $W^{u}(p)$ in $\Delta_{\varepsilon}$.

4.1.1. Expansion. If $\gamma(s)=(x(s), y(s)) \subset \Delta_{\varepsilon} \cap \mathcal{D}$ is a long admissible curve we consider the tangent vectors $\dot{\gamma}(s)$ and their images $\dot{\gamma}_{0}(s)=\operatorname{Df}(\dot{\gamma}(s))$. By Proposition $4, \dot{\gamma}_{0}$ is tangent to the stable direction $e^{(k)}$ at the point $c_{0}^{(k)}$. For this and other nearby points on $\gamma$ we can write the tangent vector

$$
\dot{\gamma}_{0}=h_{0}^{(k)} f^{(k)}+v_{0}^{(k)} e^{(k)}
$$

where $\left(f^{(k)}, e^{(k)}\right)$ is the orthogonal basis given by the most expanded and most contracted direction for $D f^{k}$ and $h_{0}^{(k)}$ and $v_{0}^{(k)}$ are the components of $\dot{\gamma}_{0}$ in this basis. Notice that the basis itself depends on the point. Proposition 2 implies that the basis varies very slowly with the base point, and Proposition 4 implies that the tangent vector $\dot{\gamma}_{0}$ is varying at positive speed with respect to this basis. We omit the calculations which are relatively standard, see for example [LV03]. Specifically this implies that the component $h_{0}^{(k)}$ of the tangent vector $\dot{\gamma}_{0}$ at some point $z_{0}=f(z) \in \gamma_{0}$ is proportional to the distance between $z$ and the critical point of order $k, c^{(k)}$. In our setting, the constants actually give

$$
\left|h_{0}^{(k)}\left(z_{0}\right)\right| \geq d\left(z, c^{(k)}\right) .
$$

We can now prove the following

Lemma 9. Suppose $\gamma \subset \Delta_{\varepsilon}$ is an admissible curve, $z \in \gamma, z_{0}=f(z) \in \mathcal{V}_{k} \backslash \mathcal{V}_{k+1}$ and $c^{(k)}$ is the critical point of order $k$ in $\gamma$. Then for a vector $w$ tangent to $\gamma$ at $z$ and all $j=0, \ldots, k$ we have

$$
\left\|D f_{z}^{j+1}(w)\right\| \geq 3^{j} d\left(z, c^{(k)}\right)\|w\|
$$

In particular

$$
\left\|D f_{z}^{k+1}(w)\right\| \geq e^{\lambda(k+1)}\|w\| .
$$

Proof. The first equality follows immediately from (15) and (9). To prove the second we need to find a bound for $d\left(z, c^{(k)}\right)$ in terms of $k$. Using the quadratic nature of $\gamma_{0}$ and the proximity to the one-dimensional map $1-a x^{2}$ with $a \approx 2$, we obtain

$$
d\left(z, c^{(k)}\right) \geq \frac{1}{3} \sqrt{d\left(z_{0}, c_{0}^{(k)}\right)}
$$

To estimate $d\left(z_{0}, c_{0}^{(k)}\right)$ we use the observation that the "real" critical value $c_{0}$ on $\gamma_{0}$, i.e. the point of tangency between $\gamma_{0}$ and the limiting stable foliation $\mathcal{E}^{(\infty)}$ lies necessarily either on $W^{s}(q)$ (this is only a possibility if $a=a^{*}$ ) or to the right of $W^{s}(q)$ in $\mathcal{Q}$. We write this as $\delta_{0}=d\left(c_{0}, W^{s}(q)\right) \geq 0$. Combining this with Lemma 5 and the rate of convergence of 
critical points of finite order $d\left(c_{0}^{k}, c_{0}\right) \leq K b^{k}$ as mentioned in Proposition 4 and taking $b$ sufficiently small, we get

$$
\begin{aligned}
d\left(z_{0}, c_{0}^{(k)}\right) & \geq d\left(z_{0}, W^{s}(q)\right)+d\left(W^{s}(q), c_{0}\right)-d\left(c_{0}^{(k)}, c_{0}\right) \\
& \geq \frac{\delta}{2} 5^{-k}+\delta_{0}-K b^{k} \geq \frac{\delta}{3} 5^{-k} .
\end{aligned}
$$

Substituting this into (16) and using the fact that we can assume $k \geq k_{0}$ as well as the definition of $k_{0}$ in (3) and of $\lambda$ in (14), we have

$$
3^{k} d\left(z, c^{(k)}\right) \geq \frac{\sqrt{\delta}}{2 \sqrt{3}}\left(\frac{3}{\sqrt{5}}\right)^{k} \geq e^{\lambda(k+1)}
$$

4.1.2. Admissibility. Returning to the proof of the Proposition, to obtain the statement about admissibility, notice first of all that combining Lemma 9 with Lemma 3 we immediately obtain the statement that if $\gamma \subset W^{u}(p) \cap \Delta_{\varepsilon}$ is admissible and $k$ is the first time that $f^{k}(\gamma) \subset \Delta_{\varepsilon}$, then $f^{k}(\gamma)$ is admissible. Now, by choosing $|b|$ and $\eta$ small we can guarantee that $W_{l o c}^{u}(p) \cap \Delta_{\varepsilon}$ is a long admissible curve. Moreover, every piece of $W^{u}(p) \cap \Delta_{\varepsilon}$ is the image of some curve in $W_{l o c}^{u}(p) \cap \Delta_{\varepsilon}$ and is therefore admissible.

4.2. Hyperbolicity after returns to $\Delta_{\varepsilon}$. Proposition 5 gives a pointwise recovery time for the hyperbolicity of points in the critical region, based on their position. The following Proposition gives a key uniformity estimate in the phase space for each parameter $a>a^{*}$.

Proposition 6. For all $a>a^{*}$ there exists a constant $N_{a}$ such that for $z \in W^{u}(p) \cap \Delta_{\varepsilon} \cap$ $\Omega(f)$, and $v$ a tangent vector to $W^{u}(p)$ at $z$, there exists $n(z) \leq N_{a}$ such that $D f_{z}^{n(z)}(v)$ is almost horizontal and

$$
\left\|D f_{z}^{n(z)}(v)\right\| \geq e^{\lambda n(z)}\|v\| .
$$

We remark that the constant $N$ is not uniformly bounded in $a$ and in particular does not apply to $a=a^{*}$. However it gives us a uniformity statement in $z$ which will implies, as we shall see below, uniform hyperbolicity for each given parameter value $a>a^{*}$. For the proof we need to extend the definition of admissibility naturally to curves which are only differentiable of class $C^{1+1}$ (Lipschitz continuous derivative).

Definition 3. We say that $\gamma(s) \subset \Delta_{\varepsilon}$ is a $C^{1+1}$ admissible curve if $|\dot{y}| /|\dot{x}|<\alpha$, and $\dot{\gamma}(s)$ is Lipschitz with Lipschitz constant $\leq \alpha$.

We also give the formal definition of a "real" critical point, which applies both to $C^{2}$ and to $C^{1+1}$ admissible curves.

Definition 4. We say that $c \in \gamma$ is a critical point if $e^{(\infty)}$ is defined at $f(c) \in \gamma$ and coincides with $D f_{c}(\dot{\gamma}(c))$.

Lemma 10. For every $a>a^{*}$, every $z \in \overline{W^{u}(p)} \cap \Delta_{\varepsilon} \cap \Omega$ lies on a $C^{1+1}$ admissible curve $\gamma$ which is the limit of $C^{2}$ admissible curves in $W^{u}(p)$ and $\gamma$ contains a unique critical point $c(\gamma)$ with $d(z, c)>0$. 
Proof. We split the proof into two parts.

Every point lies on an admissible curve. We show first of all that every point $z \in \overline{W^{u}(p)} \cap$ $\Delta_{\varepsilon} \cap \Omega$ lies on a $C^{1+1}$ admissible curve which is the limit of $C^{2}$ admissible curves in $W^{u}(p)$. Let $z \in \overline{W^{u}(p)} \cap \Delta_{\varepsilon} \cap \Omega$ and let $z_{n} \rightarrow z$ be a sequence in with $z_{n} \in W^{u}(p) \cap \Delta_{\varepsilon} \cap \Omega$. By Proposition 5 each $z_{n}$ belongs to a long admissible curve $\gamma_{n} \subset W^{u}(p)$. We can write these as functions $\gamma_{n}: I \rightarrow \mathbb{R}$ with $I=[-\varepsilon, \varepsilon]$ and suppose that converge pointwise to $\gamma: I \rightarrow \mathbb{R}$. Since $I$ is compact and $\gamma_{n}, \dot{\gamma}_{n}$ are bounded and equicontinuous sequences we have that $\gamma$ is $C^{1}$ and $\gamma_{n} \rightarrow \gamma$ in the $C^{1}$ topology. To see that $\dot{\gamma}$ is Lipschitz, let $x, y \in I$ and observe that each $\dot{\gamma}_{n}$ is a Lipschitz function with uniformly bounded Lipschitz constant $\alpha$. Then we have $\left|\dot{\gamma}_{n}(x)-\dot{\gamma}_{n}(y)\right| \leq \alpha|x-y|$ and hence $\left|\dot{\gamma}_{n}(x)-\dot{\gamma}_{n}(y)\right| \leq \alpha|x-y|$.

Every admissible curve contains a critical point. We now show that any such curve $\gamma$ contains a unique critical point. We show first that it must contain at most one, and then argue that it must contain at least one. Let $\theta\left(\gamma_{n}(t)\right)$ be the angle between the vectors $D f_{\left(t, \gamma_{n}(t)\right)}\left(1, \gamma_{n}^{\prime}(t)\right)$ and $e^{\infty}\left(f\left(t, \gamma_{n}(t)\right)\right.$. Since the image of each admissible curve is quadratic with respect to $\mathcal{E}^{(\infty)}$ we have that $\theta\left(\gamma_{n}(t)\right)$ has a strictly non-zero derivative having at most one root corresponding to a point of tangency between $f\left(\gamma_{n}\right)$ and $\mathcal{E}^{(\infty)}$. Since $\gamma_{n} \rightarrow \gamma$ in the $C^{1}$ topology, we have that $\theta(\gamma(t))$ also has strictly non-zero derivative having at most one root also corresponding to a point of tangency between $f(\gamma)$ and $\mathcal{E}^{(\infty)}$. To see that such a point exists, observe that if $a>a^{*}$ then the graph of $\gamma$ crosses the boundary of $\Delta$ twice and $f(\gamma \cap \Delta)$ is outside $\mathcal{D}$ where the foliation $\mathcal{E}^{(\infty)}$ is well defined and the extreme points of $f(\gamma \cap \Delta)$ both lie on a piece of $W^{s}(q)$ which is a leaf of the foliation $\mathcal{E}^{(\infty)}$. This implies that there exists a point outside the interior of $\mathcal{D}$ where $f(\gamma)$ is tangent to $\mathcal{E}^{(\infty)}$.

Lemma 10 allows us to define a canonical set $\mathcal{C}_{a}$ of critical points as the union of all critical points $c(\gamma)$ for every $C^{1+1}$ which are $C^{1}$ limits of long admissible curves of $W^{u} \cap \Delta_{\varepsilon}$. In the next Lemma we show that this set is bounded away from the set of nonwandering points.

Lemma 11. For all $a>a^{*}$ we have $d\left(\mathcal{C}_{a}, \Omega\right)>0$.

We emphasize that $d\left(\mathcal{C}_{a}, \Omega\right)$ is not uniformly bounded in the parameter. The constant $N_{a}$ in the Proposition will be defined below in terms of $d\left(\mathcal{C}_{a}, \Omega\right)$.

Proof. Notice first of all that $\mathcal{C}_{a} \subset \Delta_{\varepsilon}$ and thus in particular is bounded. Let $c_{k}=c\left(\gamma_{k}\right)$ be a sequence converging to some point $c$. We need to show that $c \in \mathcal{C}_{a}$. Since each $\gamma_{k}$ is the limit of long admissible curves, we can consider sequences $\gamma_{k}^{(n)} \rightarrow \gamma_{k}$ for each $k$. Using Lemma 10 and the fact that $\left\{\gamma_{k}^{(k)}\right\}$ converges pointwise to $\gamma$, we conclude that this convergence is in fact $C^{1}$. Since $\theta\left(\gamma_{k}^{(k)}\left(c_{k}\right)\right) \rightarrow 0$ we have that $\theta(\gamma(c))=0$ and this implies that $c$ is a critical point as required.

We have therefore shown that the critical set $\mathcal{C}_{a}$ is compact. Since $\Omega$ is also compact, it is sufficient to show that $\mathcal{C}_{a} \cap \Omega=\emptyset$ to imply that they are at some positive distance apart. Disjointness follows from the observation that the image of a critical point is always outside $\mathcal{D}$, while $\Omega$ is an invariant set contained in $\mathcal{D}$. 
Proof of Proposition 6. By Lemma 11 and the uniform approximation of the critical set $\mathcal{C}$ by the finite order critical sets $\mathcal{C}^{(n)}$, there exists $N_{a}$ sufficiently large so that the following two conditions hold (using also $\lambda<\log 3$ ):

$$
d\left(\mathcal{C}_{a}^{\left(N_{a}\right)}, \mathcal{C}_{a}\right)<d\left(\mathcal{C}_{a}, \Omega\right) / 2 \quad \text { and } \quad 3^{N_{a}} d\left(\mathcal{C}_{a}^{\left(N_{a}\right)}, \Omega\right) \geq e^{\lambda N_{a}}
$$

Now consider $z \in \Delta_{\varepsilon} \cap W^{u}(p) \cap \Omega$ and let $n \geq 1$ be such that $f(z) \in \mathcal{V}_{n} \backslash \mathcal{V}_{n+1}$. Recall $f\left(\Delta_{\varepsilon}\right) \subset \mathcal{V}_{k_{0}}$ and therefore such an $n$ is always well defined except for those points which map exactly to the the curve $f^{-1}\left(W_{\delta}^{s}(q)\right.$ which forms the boundary between $\mathcal{V}^{+}$and $\mathcal{V}^{-1}$. For these points we let $n=+\infty$. Then we let

$$
n(z)=\min \left\{n, N_{a}\right\} .
$$

If $n \leq N_{a}$ the statement follows from Proposition 5. Otherwise our choice of $N_{a}$ in (17) gives

$$
\left\|D f^{N_{a}}(v)\right\| \geq 3^{N_{a}} d\left(z, \mathcal{C}_{a}^{\left(N_{a}\right)}\right)\|v\| \geq 3^{N_{a}} d\left(\Omega, \mathcal{C}_{a}^{\left(N_{a}\right)}\right)\|v\| \geq 3^{N_{a}} d\left(\Omega, \mathcal{C}_{a}\right)\|v\| / 2 \geq e^{\lambda N_{a}}\|v\|
$$

The first inequality follows from Lemma 9, the second one follows from $z \in \Omega$, the third one follows from the first part of (17), and the last one follows from the second part of (17).

Finally, considering the components of $v$ in hyperbolic coordinates we have $\left\|v_{N_{a}}^{\left(N_{a}\right)}\right\| \leq$ $(b / 3)^{N_{a}}$ and $\left\|h_{N_{a}}^{\left(N_{a}\right)}\right\| \geq e^{\lambda N_{a}}$ and therefore $D f^{N_{a}}(v)$ is almost horizontal.

4.3. Uniform hyperbolicity on $W^{u}(p)$. The following Proposition is is essentially a Corollary of Proposition 6. However we state it separately as it gives an explicit construction of the constant $C_{a}$ of hyperbolicity for each $a>a^{*}$. Before stating the result we define this constant.

Let $C_{N_{a}}^{-}=\min \left\{\left\|\left(D f_{z}^{j}\right)^{-1}\right\|^{-1}: x \in \mathcal{D}, 1 \leq j \leq N_{a}\right\}$ and $C_{N_{a}}^{+}=\max \left\{\left\|D f_{z}^{j}\right\|: x \in \mathcal{D}, 1 \leq\right.$ $j \leq N_{a}$ \} denote the maximum possible contraction and the maximum possible expansion exhibited by any vector $v \in T_{x} \mathbb{R}^{2}$ for any point $x \in \mathcal{D}$ in at most $N_{a}$ iterations. Letting $C_{\varepsilon}$ denote the constant of hyperbolicity as in (6) on page 15 , we then let

$$
C_{a}=\min \left\{\frac{C_{\varepsilon}}{C_{N}^{+}}, \frac{C_{N}^{-} e^{-\lambda N}}{C_{N}^{+}}\right\}
$$

Proposition 7. For all $a>a^{*}$, all $z \in W^{u}(p) \cap \Omega(f)$ and all vectors $w$ tangent to $W^{u}(p)$ at $z$ we have

$$
\left\|D f_{z}^{n}(w)\right\| \geq C_{a} e^{\lambda n}\|w\|
$$

for all $n \geq 1$.

Proof. Let $z \in W^{u}(p) \cap \Omega(f)$ and let $w$ tangent to $W^{u}(p)$ at $z$. Since we do not assume anything about the location of $z$ the vector $w$ may or may not be almost horizontal. We distinguish these two possibilities. 
Case 1: w almost horizontal. Let $0 \leq k_{1}<\ldots<k_{s}<n$ be the sequence of returns of the iterates of $z$ to $\Delta_{\varepsilon}$ ( with $k_{1}=0$ if $z \in \Delta_{\varepsilon}$ and $k_{1}>0$ otherwise). Then for each $k_{i}$ we have an integer $n_{i}=n\left(z_{k_{i}}\right) \leq N_{a}$ given by Proposition 5 . Then we can write

$$
k_{i+1}=k_{i}+n_{i}+q_{i}
$$

where $q_{i}$ is the number of iterates during which the point remains outside $\Delta_{\varepsilon}$. From Proposition 5 properties (5) and (7), the images of the vector at these iterates remains horizontal and we have

$$
\left\|D f_{z}^{k_{i}}(w)\right\| \geq e^{\lambda k_{i}}\|w\|
$$

for all $i=1, \ldots, s$, in particular for $i=s$. If $k_{s}+n_{s} \leq n$, applying (7) to the remaining iterates gives $\left\|D f_{z}^{n}(w)\right\| \geq C_{\varepsilon} e^{\lambda n}\|w\| \geq C_{a} e^{\lambda n}\|w\|$ as required.

If $k_{s}+n_{s}>n$ we have expansion for the first $k_{s}$ iterates which gives $\left\|D f^{k_{s}}(w)\right\| \geq$ $e^{\lambda k_{s}}\|w\|$. There follow $n-k_{s} \leq n_{s} \leq N_{a}$ iterates (since $n_{s} \leq N_{a}$ ) during which we can bound the contraction coarsely by the $N_{a}$ 'th power of the maximum contraction in the region $\mathcal{D}$ which gives

$$
\left\|D f^{n}(w)\right\| \geq C_{N}^{-} e^{\lambda k_{s}}\|w\|=C_{N}^{-} e^{-\lambda N} e^{\lambda n}\|w\| .
$$

Case 2: $w$ is not almost horizontal. We now suppose that $w$ is not almost horizontal.

Claim 1. There exists

$$
N_{a} \geq m>0
$$

such that $f^{-m}(z) \in \Delta_{\varepsilon}$ and $w_{-m}=D f^{-m}(w)$ is almost horizontal.

Proof. We show first of all that some preimage of $z$ lies in $\Delta_{\varepsilon}$. Indeed, $z \in W^{u}(p)$ implies that $z_{-n} \rightarrow p$ as $n \rightarrow \infty$ and therefore that $w_{-n}$ is almost horizontal for sufficiently large $n$ since the local unstable manifold of $p$ is admissible. By the invariance of the unstable conefield outside $\Delta_{\varepsilon}$ images of $w_{-n}$ remain almost horizontal unless some return to $\Delta_{\varepsilon}$ takes place.

Now let $m>0$ be the smallest integer such that $f^{-m} \in \Delta_{\varepsilon}$. Then $w_{-m}$ is almost horizontal since every component of $W^{u}$ in $\Delta_{\varepsilon}$ is almost horizontal. From Proposition 6 it follows that $D f_{z_{-m}}^{n\left(z_{-m}\right)}\left(w_{-m}\right)$ is almost horizontal and therefore it follows necessarily that $m \leq n\left(z_{m}\right) \leq N_{a}$. Otherwise $w$ will be almost horizontal.

Returning to the proof of the Proposition, we can now argue as in the previous case to obtain exponential growth starting from time $-m$ :

$$
\left\|D f^{n}(w)\right\|=\left\|D f^{n+m}\left(w_{-m}\right)\right\| \geq C^{\prime} e^{\lambda(n+m)}\left\|w_{-m}\right\|
$$

where $C^{\prime}=\min \left\{C_{\varepsilon}, C_{N}^{-} e^{-\lambda N}\right\}$. Moreover

$$
\|w\|=\left\|D f^{m}\left(w_{-m}\right)\right\| \leq\left\|D f^{m}\right\|\left\|w_{-m}\right\| \leq C_{N}^{+}\left\|w_{-m}\right\| .
$$

Substituting this back into (18) completes the proof. 
4.4. Uniform hyperbolicity on $\Omega$. We have obtained uniform expansion estimates for vectors tangent to $W^{u}(p)$. In this section we show that these estimates can be extended to $\Omega$. This part of the argument uses very little of the specific Hénon-like form of the map and therefore we state it in a more abstract and general context.

Proposition 8. Let $f: \mathbb{R}^{2} \rightarrow \mathbb{R}^{2}$ be a $C^{1}$ diffeomorphism and $\Omega$ a compact invariant set with $|\operatorname{det} D f|<1$ on $\Omega$. Suppose that there exists some invariant submanifold $W$ dense in $\Omega$ and such that there exist constants $C, \lambda>0$ such that $\left\|D f_{z}(v)\right\| \geq C e^{\lambda n}$ for all $z \in W \cap \Omega$ and $v$ tangent to $W$. Then $\Omega$ is uniformly hyperbolic with hyperbolic constants $C$ and $\lambda$.

Proposition 8 completes the proof of part $(a)$ of the Theorem and shows that the rates of expansion and contraction admit uniform bounds independent of the parameter.

Proof. We shall show that $\Omega$ is uniformly hyperbolic by constructing an invariant hyperbolic splitting $E_{z}^{s} \oplus E_{z}^{u}$ at every point of $\Omega$ and then showing that this splitting is continuous. We carry out this construction in several steps. The starting point is the observation that $E_{z}^{u}$ is already given by the tangent direction to $W$ for all points $z \in \Omega \cap W$.

Lemma 12. For any $z \in \Omega$ and any sequence $z_{j} \in W$ with $z_{j} \rightarrow z$, the sequence $E^{u}\left(z_{j}\right)$ converges to a (unique) limit direction $E^{u}(z)$. Each vector $v \in E^{u}(z)$ satisfies

$$
\left\|D f_{z}^{n}(v)\right\| \geq C e^{\lambda n}\|v\| \quad \text { and } \quad\left\|D f_{z}^{-n}(v)\right\| \leq C^{-1} e^{-\lambda n}\|v\|
$$

for all $n \geq 1$.

Proof. Suppose $z \in \Omega$ and let $z_{j} \in W$ be a sequence with $z_{j} \rightarrow z$. Consider the sequence of corresponding directions $E^{u}\left(z_{j}\right)$. By compactness (of the space $\mathbb{S}^{1}$ of possible directions) there must exist some subsequence $z_{j_{i}}$ such that the corresponding directions $E_{j_{i}}^{u}$ converge to some direction which we call $E^{u}(z)$. Notice that a priori this direction is not unique since it depends on the choice of subsequence. We shall show first that the forward expansion and backward contraction estimates hold and then show that this actually implies uniqueness.

Let $v \in E_{z}^{u}$ and $v_{j_{i}} \in E_{z_{j_{i}}}^{u}$ be a sequence with $v_{j_{i}} \rightarrow v$. Then, for each $n \in \mathbb{N}$ we have, by the continuity of $D f^{n}$,

$$
\left\|D f_{z_{j}}^{n}\left(v_{j}\right)\right\| \rightarrow\left\|D f_{z}^{n}(v)\right\|
$$

By assumption we know that $\left\|D f_{z_{j_{i}}}\left(v_{j}\right)\right\| \geq C e^{\lambda n}\left\|v_{j}\right\|$ and therefore

$$
\left\|D f_{z}^{n}(v)\right\| \geq C e^{\lambda n}-\varepsilon
$$

for any $\varepsilon>0$. Therefore $\left\|D f_{z}^{n}(v)\right\| \geq C e^{\lambda n}$ and, since this holds for every $n$, we have the required statement as far as the expansion in forward time is concerned. To prove contraction in backward time it is sufficient to prove it for points on $W$ and then apply exactly the same approximation argument. For $z \in W$ this follows immediately from the uniform expansivity assumption in forward time. Indeed, letting $v_{-n}=D f_{z}^{-n}(v)$, the expansivity assumption gives

$$
\|v\| \geq\left\|D f_{z_{-n}}^{n}\left(v_{-n}\right)\right\| \geq C e^{\lambda n}\left\|v_{-n}\right\|
$$

which immediately implies $\left\|v_{-n}\right\| \leq C^{-1} e^{-\lambda n}\|v\|$. 
It remains to show uniqueness of $E^{u}(z)$ for each $z \in \Omega$. Suppose by contradiction that we could find two sequences $z_{j} \rightarrow z$ and $\tilde{z}_{j} \rightarrow z$ with corresponding directions $E_{z_{j}}^{u}$ and $E_{\tilde{z}_{j}}^{u}$ converging to two different directions $E_{z}^{u}$ and $\tilde{E}_{z}^{u}$. Let $v \in E_{z}^{u}$ and $\tilde{v} \in \tilde{E}_{z}^{u}$. Then $v, \tilde{v}$ must be linearly independent and thus every other vector $w \in T_{z} \mathbb{R}^{2}$ can be written as a linear combination $w=a_{1} v+a_{2} \tilde{v}$ for some $w_{1}, w_{2} \in \mathbb{R}$. By linearity and the backward contraction estimates obtained above this implies that

$$
\left\|w_{-n}\right\|=\left\|D f_{z}^{-n}(w)\right\| \rightarrow 0
$$

as $n \rightarrow \infty$. Since $w$ was arbitrary this implies that all vectors are shrinking to zero in backward time. But this is impossible since we have assumed that $|\operatorname{det} D f|<1$ and thus $\left|\operatorname{det} D f^{-1}\right|>1$ on $\Omega$.

Corollary 2. At every point $z \in \Omega$ there exists a unique tangent space splitting $E_{z}^{u} \oplus E_{z}^{s}$ which is invariant by the derivative $D f$ and which satisfies the standard uniform hyperbolicity expansion and contraction estimates.

Proof. Lemma 12 gives the expanding direction $E_{z}^{u}$ of the splitting with the required hyperbolic expansion estimates in forward time. The invariance for points in $W$ is automatic (since tangent directions to $W$ are mapped to tangent directions to $W$ ), and the invariance for general points follows immediately from the definition of $E_{z}^{u}=\lim E_{z_{j}}^{u}$, the invariance of $E_{z_{j}}^{u}$ for $z_{j} \in W$, and the continuity of $D f$.

The stable direction $E_{z}^{s}$ is given immediately by as the limit of the sequence $e^{(n)}$ of vectors most contracted by $D f_{z}^{n}$, as discussed in section 4.1. This also automatically gives the uniqueness and invariance.

To complete the proof of the Proposition, we just need to show that the given tangent space splitting is continuous. This follows by standard arguments from the uniqueness proved in Corollary 2. Indeed, for any $z \in \Omega$ and any sequence $z_{j} \in \Omega$ with $z_{j} \rightarrow z$, every limit point of the corresponding sequence of splittings $E_{z_{j}}^{u} \oplus E_{z_{j}}^{s}$ must also be a splitting $\tilde{E}_{z}^{u} \oplus \tilde{E}_{z}^{s}$. By approximation arguments identical to those used above it follows that this splitting must also satisfy the uniform hyperbolic contraction and expansion estimates. Therefore. by uniqueness, it must coincide with the existing splitting $E_{z}^{u} \oplus E_{z}^{s}$. This completes the proof that $\Omega$ is uniformly hyperbolic.

4.5. Lyapunov exponents for $f_{a^{*}}$. Finally it remains to consider the dynamics of $f_{a^{*}}$. Recall that $a^{*}$ is defined on page 8 as the first parameter for which a tangency occurs between the compact parts of $W^{s}(q)$ and $W^{u}(p)$, see Figure 11 for the pictures in the two cases $b>0$ and $b<0$.

We need to show that, for $a=a^{*}$, all Lyapunov exponents are uniformly bounded away from 0 . We show that for each point $z \in \Omega_{a^{*}}$ not in the orbit of tangency $\mathcal{T}$ (it is not necessary to consider the orbit of tangency since this is a countable set without recurrence and can therefore not support any invariant probability measure) there exists a constant $C_{z}$, a vector $v_{z}$, and a sequence $\left\{n_{i}\right\}$ with $n_{i} \rightarrow \infty$ such that, for all $i \geq 0$,

$$
\left\|D f_{z}^{n_{i}}\left(v_{z}\right)\right\| \geq C_{z} e^{\lambda n_{i}}\left\|v_{z}\right\| \text {. }
$$




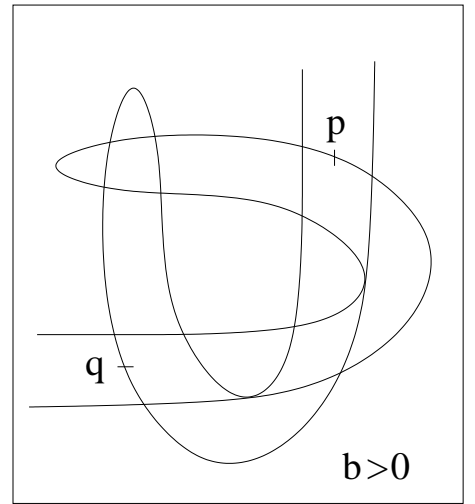

(a)

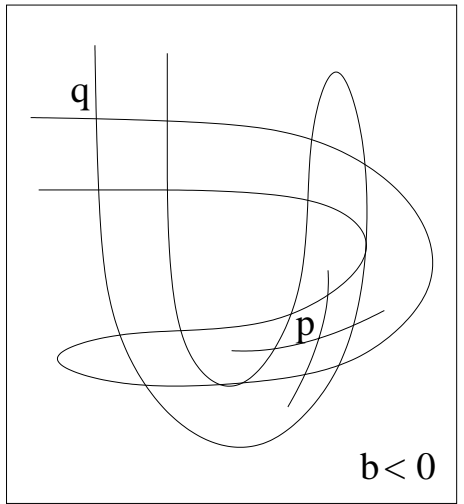

(b)

FiguRE 11. Invariant manifolds for $a=a^{*}$

This is obviously true if the orbit of $z$ never enters $\Delta_{\varepsilon}$ in forward time or it enters $\Delta_{\varepsilon}$ only a finite number of times. Indeed suppose that there exists some $k$ such that $f^{i}(z) \notin \Delta_{\varepsilon}$ for all $i \geq k$. Then let $w$ be a vector which is mapped to the horizontal vector $w_{k}=D f_{z}^{k}(w)$ after $k$ iterations. Then by (6) we have $\left\|D f_{z_{k}}^{k+n}(w)\right\| \geq C_{\varepsilon} e^{\lambda n}\left\|w_{k}\right\|$ for all $n \geq 1$. This implies that there exists a constant $C_{z}$ such that $\left\|D f_{z}^{k+n}(w)\right\| \geq C_{z} e^{\lambda(k+n)}\|w\|$ for all $n \geq 1$.

Otherwise there exists an infinite sequence $0<m_{1}<\cdots<m_{k}<\cdots$ such that $m_{k} \rightarrow \infty$ and $f^{m_{k}}(z) \in \Delta_{\varepsilon}$. By Lemma 10, $z_{m_{i}}=f^{m_{i}}(z)$ lies on either a $C^{2}$ long admissible curve or a $C^{1+1}$ long admissible curve which is the $C^{1}$ limit of $C^{2}$ long admissible curves in $W^{u}(p)$. Since $z$ has an infinite number of returns to $\Delta_{\varepsilon}$, this implies in particular that $z \notin W^{s}(q)$ and so $z_{m_{i}} \notin W^{s}(q)$ and so there exists $n_{i}=n\left(z_{m_{i}}\right)$ such that $f\left(z_{m_{i}}\right) \in \mathcal{V}_{n_{i}} \backslash \mathcal{V}_{n_{i}+1}$. Therefore exactly the same arguments as in Lemmas 9 and 12 show that for a vector $v_{i}$ tangent to such an admissible curve $\gamma$ at $z_{m_{i}}$ we have

$$
\left\|D f_{z_{m_{i}}}^{n_{i}+1}\left(v_{i}\right)\right\| \geq e^{\lambda\left(n_{i}+1\right)}\left\|v_{i}\right\| \text {. }
$$

Notice that since the $C^{1}$ limits of $C^{2}$ admissible curves are unique, as proved above, we have $v_{i+1}=D f^{m_{i+1}-m_{i}}\left(v_{i}\right)$. Then, by (6) and (19) we have

$$
\left\|D f^{m_{i}+n_{i}+1-m_{1}}\left(v_{1}\right)\right\| \geq e^{\lambda\left(m_{i}+n_{i}+1-m_{1}\right)}\left\|v_{1}\right\| \text {. }
$$

Then we can define $v_{z}=D f^{-m_{1}}\left(v_{1}\right)$ and we have $\left\|D f^{m_{i}+n_{i}+1}\left(v_{z}\right)\right\| \geq C_{z} e^{\lambda\left(m_{i}+n_{i}+1\right)}\left\|v_{z}\right\|$. where the constant $C_{z}$ is required simply to compensate for the possible lack of expansion for the first $n_{1}$ iterates. In particular it can be chosen by considering the maximum possible contraction along the orbit of $z$ for the first $n_{1}$ iterations

$$
C_{z}=\min _{\|v\|=1}\left\|D f_{z}^{n_{1}}(v)\right\| .
$$

We have shown therefore that for each $z \in \Omega \lim _{\sup _{n \rightarrow \infty}} \frac{1}{n} \ln \left\|D f_{z}^{n}\right\| \geq \lambda$. This clearly implies the same bound for the limit wherever it exists. In particular any point which is typical for some ergodic invariant probability measure and for which therefore such a limit does exist, will have a positive Lyapunov exponent $\geq \lambda$. By dissipativity this immediately 
implies also that the other Lyapunov exponent is negative and uniformly bounded away from 0 both in the dynamical and in the parameter space.

\section{REFERENCES}

[Bed05] Eric Bedford (2005), Personal communication.

[BS02] Eric Bedford and John Smillie, Polynomial diffeomorphisms of $\mathbf{C}^{2}$. VIII. Quasi-expansion, Amer. J. Math. 124 (2002), no. 2, 221-271.

[BS04] , Real polynomial diffeomorphisms with maximal entropy: tangencies, Annals of Math. 160 (2004), 1-26.

[BS06] _ Real Polynomial Diffeomorphisms with Maximal Entropy: II. Small Jacobian, Erg. Th. \& Dyn. Syst. 26 (2006), no. 5, 1259-1283.

[BC91] M. Benedicks and L. Carleson, The dynamics of the Heńon map, Ann. of Math. 133 (1991), 73-169.

[BV01] Michael Benedicks and Marcelo Viana, Solution of the basin problem for Hénon-like attractors, Invent. Math. 143 (2001), no. 2, 375-434.

[BY00] Michael Benedicks and Lai-Sang Young, Markov extensions and decay of correlations for certain Hénon maps, Astérisque (2000), no. 261, xi, 13-56 (English, with English and French summaries). Géométrie complexe et systèmes dynamiques (Orsay, 1995).

[BY93] _ Sinaŭ-Bowen-Ruelle measures for certain Hénon maps, Invent. Math. 112 (1993), no. 3, 541-576.

[CLR06] Yongluo Cao, Stefano Luzzatto, and Isabel Rios, A Non-hyperbolic system with strictly nonzero Lyapunov exponents for all invariant measures, Disc. \& Cont. Dyn. Syst. 15 (2006), no. 1, $61-71$.

[DN79] R. Devaney and Z. Nitecki, Shift automorphisms in the Hénon mapping, Comm. Math. Phys. 67 (1979), no. 2, 137-146.

[Hén76] M. Hénon, A two dimensional mapping with a strange attractor, Comm. Math. Phys. 50 (1976), $69-77$.

[HL06] Mark Holland and Stefano Luzzatto, Stable manifolds under very weak hyperbolicity conditions, J. Differential Equations 221 (2006), no. 2, 444-469.

[LV03] Stefano Luzzatto and Marcelo Viana, Parameter exclusions in Hénon-like systems, Russian Mathematical Surveys 58 (2003), no. 6, 1053-1092.

[dMvS93] Welington de Melo and Sebastian van Strien, One-dimensional dynamics, Ergebnisse der Mathematik und ihrer Grenzgebiete (3) [Results in Mathematics and Related Areas (3)], vol. 25, Springer-Verlag, Berlin, 1993.

[MV93] Leonardo Mora and Marcelo Viana, Abundance of strange attractors, Acta Math. 171 (1993), no. $1,1-71$.

[Ose68] V. I. Oseledec, A multiplicative ergodic theorem. Characteristic Ljapunov, exponents of dynamical systems, Transactions of the Moscow Mathematical Society 19 (1968).

[PdM82] J. Palis and W. de Melo, Geometric Theory of Dynamical Systems, An Introduction, Springer, 1982.

[YP76] Ya. Pesin, Families of invariant manifolds corresponding to non-zero characteristic exponents, Math. USSR. Izv. 10 (1976), 1261-1302.

[Pes77] Ya. B. Pesin, Characteristic Lyapunov exponents and smooth ergodic theory, Russian Math. Surveys 324 (1977), 55-114.

[WY01] Qiudong Wang and Lai-Sang Young, Strange attractors with one direction of instability, Comm. Math. Phys. 218 (2001), no. 1, 1-97. 
Department of Mathematics, Suzhou University, Suzhou 215006, Jiangsu, P.R. China E-mail address: ylcao@suda.edu.cn, yongluocao@yahoo.com

Dept. of Mathematics, Imperial College, 180 Queen's Gate, London SW7 2AZ, UK E-mail address: Stefano.Luzzatto@imperial.ac.uk URL: http://www.ma.ic.ac.uk/ luzzatto

Universidade Federal Fluminense, Niteroi, RJ, Brazil.

E-mail address: rios@mat.uff.br 